This is the final peer-reviewed accepted manuscript of:

A. Trotta, M. D. Felice, F. Montori, K. R. Chowdhury and L. Bononi, "Joint Coverage, Connectivity, and Charging Strategies for Distributed UAV Networks," in IEEE Transactions on Robotics, vol. 34, no. 4, pp. 883-900, Aug. 2018.

The final published version is available online at :
http://dx.doi.org/10.1109/TRO.2018.2839087

Rights / License:

The terms and conditions for the reuse of this version of the manuscript are specified in the publishing policy. For all terms of use and more information see the publisher's website. 


\title{
Joint Coverage, Connectivity and Charging Strategies for Distributed UAV Networks
}

\author{
Angelo Trotta*, Marco Di Felice*, Federico Montori*, Kaushik R. Chowdhury ${ }^{\dagger}$, Luciano Bononi* \\ * DISI, University of Bologna, Italy \\ $\dagger$ Northeastern University, Boston, USA \\ Emails: \{angelo.trotta5, marco.difelice3, federico.montori2, luciano.bononi\}@unibo.it, krc@ece.neu.edu
}

\begin{abstract}
This paper proposes deployment strategies for consumer Unmanned Aerial Vehicles (UAVs) to maximize the stationary coverage of a target area and to guarantee the continuity of the service through energy replenishment operations at ground charging stations. The three main contributions of our work are: (i) A centralized optimal solution is proposed for the joint problem of UAV positioning for a target coverage ratio and scheduling the charging operations of the UAVs that involves travel to the ground station. (ii) A distributed game theorybased scheduling strategy is proposed using normal-form games with rigorous analysis on performance bounds. Further, a bioinspired scheme using attractive/repulsive spring actions are used for distributed positioning of the UAVs. (iii) The cost-benefit tradeoffs of different levels of cooperation among the UAVs for the distributed charging operations is analyzed. Our work demonstrates that the distributed deployment using only 1-hop messaging achieves approximation of the centrally computed optimum, in terms of coverage and lifetime.
\end{abstract}

Index Terms-Aerial Robotics, Force Control, Networked Robots, Sensor Networks, Battery Recharge Scheduling, Game Theory

\section{INTRODUCTION}

$\mathbf{U}$ NMANNED AERIAL VEHICLES (UAVs) represent one of the fastest growing technological sectors today, with over $126 \%$ annual increase in market size in 2016 and estimated global revenues of around 3 billion dollars [1]. Such UAVs are being envisaged for a variety of use-cases like disaster recovery [5] and cellular data offloading [6], which require a connected aerial mesh network that provides continuous spatio-temporal coverage of a target area [2][3][4]. Two key issues must be addressed to facilitate the deployment of the UAV mesh network: (i) multi-objective localization of UAVs that considers the sensing coverage needs of the network, and (ii) maintaining persistent service considering energyrelated interruptions. Our work tackles these issues through a rigorously derived analytical framework that provides both centralized and distribution solutions.

\section{A. Challenges in UAV localization and persistent service}

The central concern in multi-objective localization is how to set the static locations of each UAV in a 3D space, so that both the sensing needs of the application and the aerial mesh connectivity requirements are met [7]. While several works have looked into communication-aware mobility schemes for UAVs, few of them jointly address the problem of static coverage [8][9][11].
The second issue of ensuring persistent service stems from the limitations of the on-board battery, which is in the order of fraction of an hour (typically 15-20 mins) for most commercial consumer-grade UAVs [12]. Solutions for terrestrial technology like cross-layer energy-efficient communication, when mapped to UAVs, are unlikely to make a significant impact [15]. This is because the ratio of the cumulative energy cost of all sensing/communication tasks to that of operating the motors is $20: 80$ [16]. We believe these practical difficulties can be surmounted by carefully scheduling energy replenishment operations and leveraging charging stations on the ground. To realize this paradigm, an intelligent scheduler policy is needed that directs the UAVs to ground-based charging stations depending on the application requirements and the scenario constraints (e.g. number of UAVs which can charge synchronously). Previous research on the UAV scheduling problem has revealed that the problem is NPcomplete [2][3][18], and with the additional consideration of ensuring coverage, the complexity of the problem only increases.

\section{B. Proposed Research and Contributions}

We approach the combined problem of stationary UAV coverage and energy replenishment scheduling by proposing a combined framework which ensures that user-defined coverage metrics are met and mesh connectivity is maintained, while maximizing the persistent service requirement. We consider a generalized system model, composed of $N_{S}$ UAVs and one ground-based charging station to make the following three main research contributions:

- We devise optimal and heuristic solutions to both the problems of UAV positioning and scheduling the recharging cycles considering also the impact of unique issues related to UAVs, like their height above ground, the energy overhead of making the ascent/descent, and the beaconing frequency.

- We develop a distributed UAV deployment algorithm building on virtual spring mechanisms [9], which relies on local information (e.g. residual energy), and the information received by other UAVs forming the aerial mesh. We compare this approach with the global optimal found through centralized knowledge.

- We investigate the cost-benefit tradeoff of information exchange among UAVs for ensuring persistence service 
that maximizes network lifetime. Then, we formulate three variants of game-theory based UAV energy cycling schedulers with varying levels of information exchange performed by the UAVs, and compute the mixed strategies guaranteeing the Nash equilibrium for them. Importantly, we show that with only local 1-hop knowledge our distributed scheduler performs close to that with global multi-hop knowledge with a centralized scheduler, which has many practical deployment advantages.

The rest of the paper is structured as follows. We review the state of the art addressing coverage and energy-efficiency issues in Section II. The system model and problem formulation are described in Section III. The optimal solution through global coordination is presented in Section IV. The distributed approaches for charging scheduling and UAV positioning are detailed in Section V. A rigorous performance evaluation is presented in Section VI and Section VII concludes the paper.

\section{RELATED WORKS}

We review existing works separately for the dual topics of (i) UAV positioning for stationary coverage and (ii) UAV lifetime/energy management.

\section{A. UAV positioning for stationary coverage}

Compared to classical multi-robot coverage problems, a major novelty in centralized coverage schemes is given by the possibility to control the altitude of each UAV. In [21], the authors investigate the optimal 3D placement of UAVs, so that the number of connected users on the ground is maximized, while the transmitting power of the UAVs is minimized. Similarly, the study in [8] derives fundamental results about 3D coverage, clarifying the relationship between altitude, beamwidth of the antenna and coverage probability. The tradeoff between coverage and connectivity is investigated in [22], where the authors show that guaranteeing both these goals can be challenging in highly sparse networks. Considering distributed approaches, [9] and [24] aim to achieve maximum stationary coverage of a target scenario while preserving connectivity among the UAVs. More specifically, [9] proposes a mobility scheme based on the virtual spring model [11], such that all the aerial links experience the same Quality of Service (QoS) regardless of the propagation conditions. A channelaware swarm mobility scheme is proposed in [24], based on the cluster-breathing technique and on the utilization of the Receiver Signal Strength (RSS) metric as proxy of the link quality. The problem of minimizing the total distance travelled by the mobile robots in order to visit a set of target locations, denoted as target assignment in robotic networks, is address by [10]. For the link communications, the authors used both a simple circular range-based model and a region-based model in which all robots within the same region can communicate with each other.

\section{B. UAV lifetime/energy management}

UAV network lifetime maximization can be achieved through different approaches, i.e. energy-aware control proto- cols, energy-aware network protocols, and external energy replenishment through ground-based charging stations. Energyaware control protocols minimize the unnecessary maneuvers of mobile devices [7], or devise an energy-aware path plan meeting constraints on minimal coverage [23]. Energy-aware network protocols for UAVs mitigate the impact of wireless network operations on the battery consumption, with a comprehensive survey available in [15].

For the solutions involving energy replenishment through scheduling of UAVs, we further distinguish between two classes, i.e. (i) path planning-oriented, and (ii) stationary coverage-oriented. In (i), the UAVs keep flying over a set of sites, and the goal of the scheduler is to determine the optimal tour of the UAVs, so that each site is visited with a given frequency. Examples are described in [2], [18], [25], [26], [28] and [29]. More specifically, the authors of [26] formulate the scheduling problem by means of temporal logic, while the scheduler in [18] computes the itinerary of each UAVs, so that the presence of an energy-feasible path toward the replenishment station is always guaranteed. In [2], the paths of UAVs are computed in order to fully cover the trajectory of a mobile user; to this aim, the scheduler assigns each UAV to a space-time segment minimizing the travel distance. The charging slots are allocated via Mixed Linear Integer Programming (MILP) techniques. In [28] and [29], the authors investigate the utilization of Unmanned Ground Vehicles (UGVs) as mobile recharging stations and they develop path planning algorithms for both the UAVs and the UGVs, by considering a modified version of the Traveling Salesman Problem.

Stationary coverage-oriented schedulers assume the presence of UAVs at fixed positions, and aim to guarantee the continuous coverage of a target area. The authors of [3], prove that the problem of determining the minimum number of UAVs guaranteeing the persistent coverage of a target area is NPcomplete. The study in [13] describes a network architecture composed of fixed battery-powered Access Points (APs) and of UAVs, which carry full-charged batteries back and forth between the APs and an energy-supplying station. In this case, the static coverage is provided by the wireless ground mesh formed by the APs, while the UAVs perform the energy replenishment operations. Similar to [3], we address stationary coverage. However, the number of UAVs is assumed as an input to the problem in our case, and the goal of the scheduler is to determine the maximum lifetime while guaranteeing a minimum coverage. Hence, the problem becomes computationally tractable and closer to the characteristics of a real scenario, where the available resources (e.g. the number of the UAVs) are typically known in advance.

On a more technology development front, the study in [17] demonstrates a guidance system enabling the UAV to land on a charging station. The problem of a reliable recharging process is addressed in [14], where the authors design a ground recharge station for UAVs, and propose a charging scheduler which assign priorities to UAVs in proportion to their battery level. The authors of [27], describe a battery replacement system for UAVs, evaluating it on a small test-bed. In [30] the authors describe the design of an autonomous battery change/recharge station, and demonstrates the possibility to 
support persistent missions of more than 3 hours duration with small UAVs.

The current work extends our previous conference paper [4], in which we introduced a preliminary version of the problem and the centralized deployment. Here, we considerably enhance the system model and problem formulation, and also design the distributed deployment. The performance evaluation has also been completely revised to reflect these new contributions.

\section{SYSTEM MODEL}

We now introduce the system model and the assumptions adopted in the rest of the paper, followed by a mathematical definition of the research problem. Table I list the symbols and variables for ease of reference.

TABLE I

TABLE OF VARIABLES/SYMBOLS

\begin{tabular}{|c|c|}
\hline$D$ & Area to cover \\
\hline$N_{S}$ & Numbers of UAVs \\
\hline$A=\left\{a_{1}, \ldots, a_{N_{s}}\right\}$ & Set of the UAVs \\
\hline$T=\left\{t_{0}, t_{1}, \ldots\right\}$ & Slotted time \\
\hline$t_{\text {slot }}$ & Length of a time slot \\
\hline$S_{E}$ & Charging station \\
\hline$h$ & Flight altitude \\
\hline$\theta$ & Angle of the sensing cone \\
\hline$\alpha_{t}$ & Per-slot Loss of energy while flying \\
\hline$\beta_{t}$ & Per-slot Gain of energy while recharging \\
\hline$\gamma_{h}$ & Loss of energy for the descending operation \\
\hline$\delta_{h}$ & Loss of energy for the ascending operation \\
\hline$O P(h)=\gamma_{h}+\delta_{h}$ & Energy overhead for the to-and-fro journey \\
\hline$\phi: T \rightarrow\{0,1\}$ & Availability function for the station $S_{E}$ \\
\hline$S=\left\{s_{\mathrm{fly}}, s_{\mathrm{rec}}\right\}$ & Set of possible UAV states \\
\hline$E: A, T \rightarrow \mathbb{R}$ & Residual energy function \\
\hline$s: A, T \rightarrow\{0,1\}$ & Recharge scheduling function \\
\hline$G_{\mathrm{OK}}$ & Action of attempting to access the station $S_{E}$ \\
\hline$G_{\mathrm{NO}}$ & Action of remaining in flight state \\
\hline$R_{\mathrm{OK}}$ & Action of releasing the station $S_{E}$ \\
\hline$R_{\mathrm{NO}}$ & Action of not releasing the station $S_{E}$ \\
\hline$p_{G}^{i, j}$ & $\begin{array}{l}\text { Probability that UAV } a_{i} \text { at time slot } t_{j} \\
\text { executes the action } G_{\mathrm{OK}}\end{array}$ \\
\hline$p_{R}^{k, j}$ & $\begin{array}{l}\text { Probability that UAV } a_{k} \text { at time slot } t_{j} \\
\text { executes the action } R_{\mathrm{OK}}\end{array}$ \\
\hline$p_{B}^{i, j}$ & $\begin{array}{l}\text { Probability that UAV } a_{i} \text { at time slot } t_{j} \\
\text { finds the station } S_{E} \text { busy }\end{array}$ \\
\hline$p_{T_{0}}^{k, j}$ & $\begin{array}{l}\text { Probability that no UAV, except for } a_{k} \text {, } \\
\text { at time slot } t_{j} \text { executes the action } G_{\mathrm{OK}}\end{array}$ \\
\hline
\end{tabular}

\section{A. Scenario modeling}

We consider a square area of size $D \mathrm{~m}^{2}$, and a set $A=$ $\left\{a_{1}, a_{2}, \ldots, a_{N_{s}}\right\}$ of $N_{s}$ UAVs. Each UAV is able to sense the

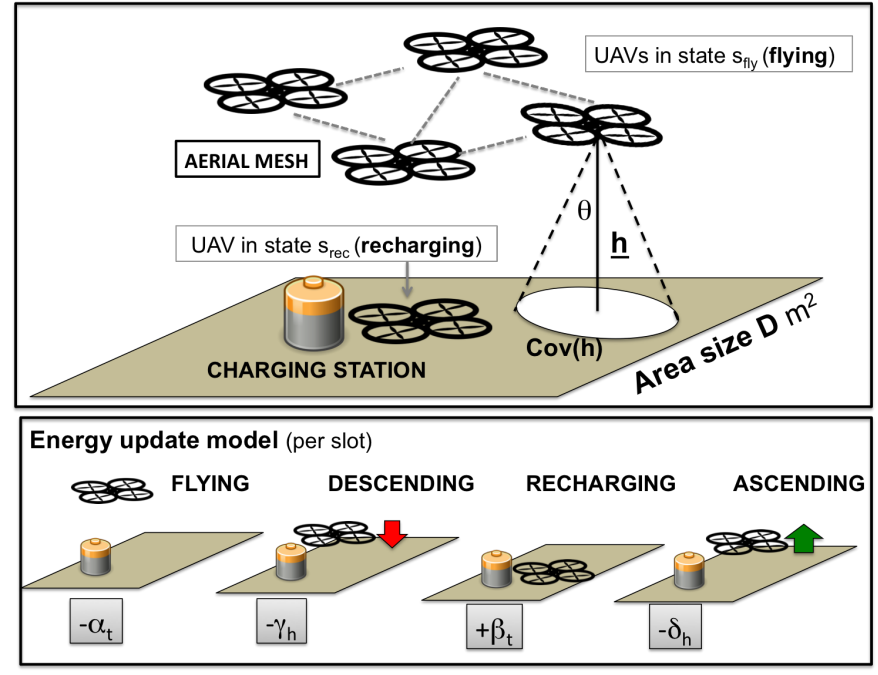

Fig. 1. The aerial mesh network with the charging station on the ground.

environment, communicate wirelessly with other peers, and recharge its battery at the replenishment station $S_{E}$ that is located on the ground at the center of the scenario. We assume that the station can dispense energy at a speed of $C_{S_{E}}[\mathrm{~J} / \mathrm{sec}]$, while each UAV $a_{i}$ has a maximum battery capacity equal to $E^{\mathrm{MAX}}$. The UAVs form a connected network at $h$ meters from the ground. Depending on $h$, each UAV is able to sense an area $\operatorname{Cov}(h)$ equal to:

$$
\operatorname{Cov}(h)=\pi \cdot\left(h \cdot \tan \left(\frac{\theta}{2}\right)\right)^{2}
$$

where $\theta$ is the angle of the sensing cone depicted in Figure 1.

Without loss of generality, we assume that the time is divided into consecutive time slots $T=\left\{t_{0}, t_{1}, \ldots\right\}$ of length equal to $t_{\text {slot. }}$. We denote with $E\left(a_{i}, t_{j}\right)$ the residual energy of agent $a_{i} \in A$ at time slot $t_{j} \in T$. We assume that all the UAVs start with the same energy amount, equal to $E_{\text {init }}$, i.e. $E\left(a_{i}, t_{0}\right)=E_{\text {init }} \leq E^{\mathrm{MAX}}, \forall a_{i} \in A$. Next, we introduce the function $\phi: T \rightarrow\{0,1\}$ indicating the availability of the replenishment station at a given time slot. More specifically, $\phi\left(t_{j}\right)=0$ indicates that the station is occupied by one UAV at slot $t_{j}$, while $\phi\left(t_{j}\right)=1$ indicates that the station is currently available. At each slot $t_{j}$, each UAV $a_{i} \in A$ can be in one of the following two states:

- State $s_{\text {fly }}$ (flying): the UAV $a_{i}$ does not use the station $S_{E}$, losing a per-slot constant amount of energy while flying (denoted as $\alpha_{t}$ in the following).

- State $s_{\text {rec }}$ (recharging): the UAV $a_{i}$ recharges its battery on the ground, gaining a per-slot constant amount of energy (denoted as $\beta_{t}$ in the following).

Let $S=\left\{s_{\mathrm{fly}}, s_{\mathrm{rec}}\right\}$ denote the UAV state set. Based on its state at time slot $t_{j-1}$, each UAV can execute different actions at slot $t_{j}$. More specifically, if UAV $a_{i}$ is in state $s_{\text {fly }}$ at time slot $t_{j-1}$, then one between the following two actions can be selected:

- $\boldsymbol{G o}\left(G_{\mathrm{OK}}\right)$ - The UAV $a_{i}$ attempts to access the replenishment station. If the station $S_{E}$ is free then the UAV changes its state to $s_{\text {rec }}$, otherwise it remains in state $s_{\text {fly }}$. 
- Stay $\left(G_{\mathrm{NO}}\right)$ - The UAV $a_{i}$ remains in state $s_{\mathrm{fly}}$. Similarly, if UAV $a_{i}$ is in state $s_{\text {rec }}$ at time slot $t_{j-1}$, then one of the following two actions is selected:

- Release $\left(R_{\mathrm{OK}}\right)$ - The UAV $a_{i}$ releases the replenishment station and changes its state to $s_{\text {fly }}$.

- Keep $\left(R_{\mathrm{NO}}\right)$ - The UAV $a_{i}$ remains in state $s_{\text {rec }}$ and keeps recharging for another slot.

The action of trying to acquire the replenishment station has a cost of $\gamma_{h}=\gamma \cdot h$, which models the energy overhead for the descent operations, i.e. from landing to the ground from an initial height of $h$. Similarly, the action of releasing the station, and of flying back to the aerial mesh, has a cost of $\delta_{h}=\delta \cdot h$. We assume that the power consumed during horizontal flight and hover is approximately equivalent [31] and modeled by the parameter $\alpha_{t}$. Furthermore, since a single replenishment station is assumed, we do not expect to see large distance between the station and the peripheral UAVs, and hence we assume that values of $\gamma_{h}$ and $\delta_{h}$ will depend on the flight altitude $h$ only: the $\gamma$, and $\delta$ parameters will then correspond to the average of the energy power consumption during the descending and ascending operations, regardless of the length of the path that the UAVs need to travel before reaching the target position. In the following, we indicate with $O P(h)=$ $\gamma_{h}+\delta_{h}$ the total energy overhead for completing the to-andfro journey. We define $\alpha_{t}=\alpha \cdot t_{\text {slot }}$ as the amount of energy loss during an entire time slot while being in state $s_{\mathrm{fly}}$ and $\beta_{t}=\beta \cdot t_{\text {slot }}$ as the amount of energy gained during an entire time slot while being in state $s_{\text {rec }}$. Selection of $\alpha, \beta, \gamma$ and $\delta$ parameters depends on the specifications of the hardware in use, and we list later their quantitative representations for our use-case.

\section{B. Problem formulation}

We denote with $s\left(a_{i}, t_{j}\right): A, T \rightarrow\{0,1\}$ the scheduling function that defines the state for each UAV $a_{i} \in A$ at each time slot $t_{j} \in T$. More specifically, if $s\left(a_{i}, t_{j}\right)=1$ then the UAV $a_{i}$ is in state $s_{\text {fly }}$ at time slot $t_{j}$; vice-versa, if $s\left(a_{i}, t_{j}\right)=$ 0 , then the UAV $a_{i}$ is in state $s_{\text {rec }}$ at time slot $t_{j}$.

Let $g\left(a_{i}, t_{j}\right): A, T \rightarrow\{0,1\}$ be the function indicating whether the UAV $a_{i}$ executes or not the action $G_{\mathrm{OK}}$ at the beginning of time slot $t_{j}$. Clearly, $g\left(a_{i}, t_{j}\right)=1$ requires that $s\left(a_{i}, t_{j-1}\right)=1$. Let $r\left(a_{i}, t_{j}\right): A, T \rightarrow\{0,1\}$ be the function indicating whether the agent $a_{i}$ executes or not the action $R_{\mathrm{OK}}$ at the beginning of time slot $t_{j}$. Again, $r\left(a_{i}, t_{j}\right)=1$ requires that $s\left(a_{i}, t_{j-1}\right)=0$. The $E\left(a_{i}, t_{j}\right)$ function is updated according to the following Equation:

$$
\begin{aligned}
E\left(a_{i}, t_{j}\right)=E\left(a_{i}, t_{j-1}\right) & -s\left(a_{i}, t_{j}\right) \cdot \alpha_{t} \\
& +\left(1-s\left(a_{i}, t_{j}\right)\right) \cdot \beta_{t} \\
& -g\left(a_{i}, t_{j}\right) \cdot\left(\gamma_{h}+\delta_{h} \cdot\left(1-\phi\left(t_{j}\right)\right)\right) \\
& -r\left(a_{i}, t_{j}\right) \cdot \delta_{h}
\end{aligned}
$$

Here, $E\left(a_{i}, t_{j-1}\right)$ is the residual energy for agent $a_{i}$ at the previous time slot. The second term refers to the energy consumption for flying; the third term refers to the energy gained while recharging; the last two terms refer to the energy lost when attempting to change the current state.

We are interested in deploying an energy-efficient UAV network, with constraints in terms of the area covered and of the persistence in service. Let $\kappa$ be a system threshold on the fraction of the area covered by the aerial network. Our Constrained Coverage and Persistence Aerial Network Deployment (CCPANP) problem can be informally defined as: how to determine an optimal charging scheduling function $s\left(a_{i}, t_{j}\right), \forall a_{i} \in A, \forall t_{j} \in T$, so that: $(i)$ the fraction of area covered by the UAV network at slot $t_{j}$ is always greater than $\kappa$, and (ii) the network lifetime is prolonged as much as possible. Formally, the CCPANP problem is defined as:

Definition 1 (CCPANP problem). Let $t_{\text {final }}$ be the lifetime of the system defined by the smallest time slot $t_{j} \in T$ where $\exists a_{i} \in A$ such that $E\left(a_{i}, t_{j}\right)=0$, i.e. the UAV $a_{i}$ runs out of battery. Given the set of UAVs $A=\left\{a_{1}, a_{2}, \ldots, a_{N_{s}}\right\}$ and the factors $\alpha, \beta, \gamma, \delta$, we want to determine the optimal $s\left(a_{i}, t_{j}\right)$ function $\forall a_{i} \in A, \forall t_{j} \in T$ such that the network lifetime $t_{\text {final }}$ is maximized and the following constraints are met, $\forall a_{i} \in$ $A, \forall t_{j} \in T$ :

$$
\begin{aligned}
& \sum_{a_{i} \in A}\left(1-s\left(a_{i}, t_{j}\right)\right)=1 \quad \forall t_{j} \in T \\
& E\left(a_{i}, t_{j}\right)>0, E\left(a_{i}, t_{j}\right) \leq E_{a_{i}}^{\mathrm{MAX}} \quad \forall a_{i} \in A, \forall t_{j}<t_{\text {final }} \\
& \rho_{j}=\frac{C\left(t_{j}\right)}{D} \geq \kappa \quad \forall t_{j} \in T
\end{aligned}
$$

Here, the first condition asserts that at each time slot $t_{j}$ only one UAV can utilize the station $S_{E}$. The second condition asserts that no UAV can run out of battery till $t_{\text {final }}$. The last constraint asserts that the ratio $\rho_{j}$ between the area covered at time $t_{j}$ by the aerial network, i.e. $C\left(t_{j}\right)$, and the total area to cover, i.e. $D$, is greater then a given system threshold $\kappa$. $C\left(t_{j}\right)$ is defined as:

$$
C\left(t_{j}\right)=\bigcup_{i=1}^{N_{s}}\left(\operatorname{Surf}\left(t_{j}, a_{i}, h, \theta\right) \cdot s\left(a_{i}, t_{j}\right)\right)
$$

where $\operatorname{Surf}\left(t_{j}, a_{i}, h, \theta\right)$ is the surface covered at time slot $t_{j}$ by the UAV $a_{i}$ flying at altitude $h$ with the sensor angle $\theta$. Clearly, $\operatorname{Surf}\left(t_{j}, a_{i}, h, \theta\right)=\emptyset$ if $s\left(a_{i}, t_{j}\right)=0$, i.e. the UAV $a_{i}$ is in state $s_{\text {rec }}$ at time slot $t_{j}$.

\section{Centralized Optimal Approach}

In this Section, we provide the optimal solution to the CCPANP research problem defined above. First, we observe from [34] that the optimal coverage is achieved when placing the UAVs in regular hexagon patterns with side length equal to $R=h \cdot \tan \left(\frac{\theta}{2}\right)$, where $R$ is the area coverage radius. The separation between the UAVs is hence equal to $R \cdot \sqrt{3}$. Let $N_{\min }$ be the minimum number of UAVs that is required to guarantee at time slot $t_{j}$ that $\rho_{j} \geq \kappa$. Using results in [34], we compute $N_{\min }$ as follows:

$$
N_{\min }=\left\lceil\frac{\kappa \cdot D}{\left(h \cdot \tan \left(\frac{\theta}{2}\right)\right)^{2} \cdot \frac{3 \cdot \sqrt{3}}{2}}\right\rceil
$$




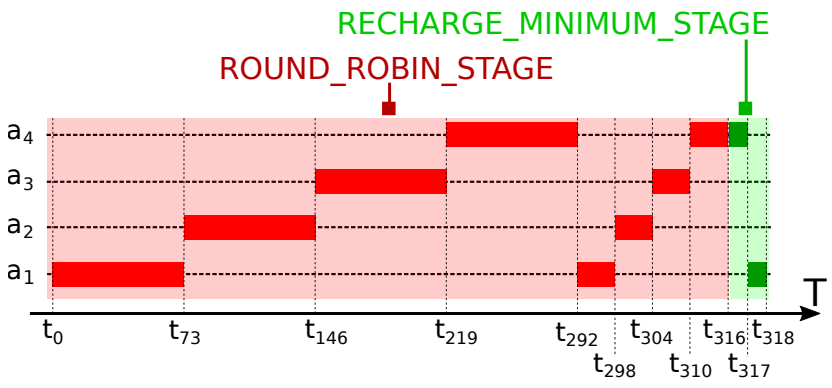

(a) Charging sequence

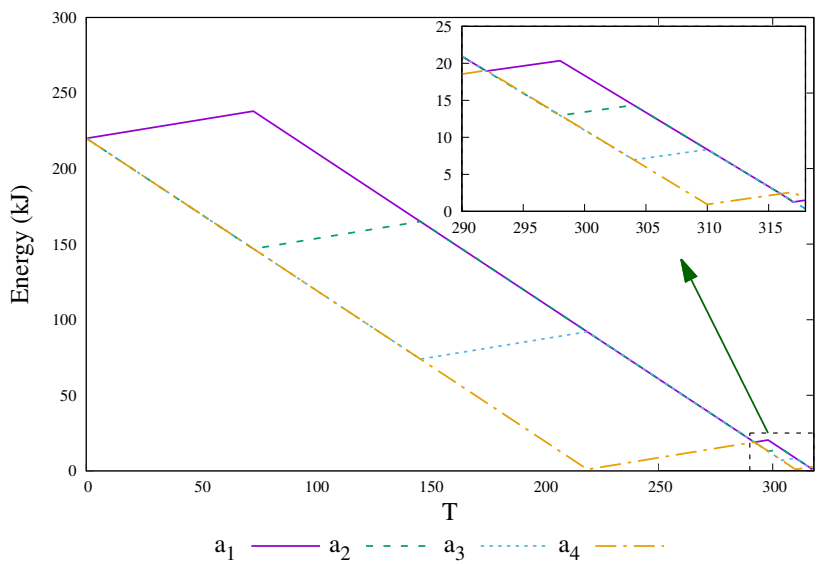

(b) UAVs energy levels

Fig. 2. The two stages of Algorithm 1 with $N_{S}=4, E_{\text {init }}=$ $220 k J, O P(h)=200 J, \alpha_{t}=1000 J, \beta_{t}=250 J$. In 2(a) the bars define the time spent by the UAV $a_{i}$ on the recharging station $S_{E}$. The ROUND_ROBIN_STAGE is drawn in red, while the RECHARGE_MINIMUM_STAGE is drawn in green. In 2(b) we show the UAV energy levels during the charging operations.

Given (7), we assume that $N_{S} \geq N_{\min }+1$ in order to be able to cover the requested area of size $\kappa \cdot D \mathrm{~m}^{2}$.

Algorithm 1 shows the pseudo-code for solving the $\mathrm{CC}$ PANP problem. We assume that all the UAVs have the same initial amount of energy, indicated as $E_{\text {init }}$. At each time-slot, the schedule method is executed, and the UAV with id equal to currentCharge is charged. Moreover, we check that all UAVs have energy greater than zero, otherwise the algorithm ends (at line 4). The algorithm can work in two stages, respectively the ROUND_ROBIN_STAGE, which can be repeated over several iterations, and the RECHARGE_MINIMUM_STAGE. In Figure 2(a) and 2(b), we show a graphical example of these two stages.

In the ROUND_ROBIN_STAGE, we let each UAV $a_{i}$ recharge of the maximum number of sequential slots, denoted as roundSize $[i]$. The exact value of roundSize[i] is computed by the allocateRoundCharge method by: (i) considering the UAV with maximum residual energy (line 46); (ii) computing the maximum number of slots before such node will drain its energy (numRoundsPer $U A V$ ); (iii) assigning numRoundsPerUAV to all the UAVs (lines 59-61); (iv) in case of residuals, allocate the extraRounds slots to the UAVs with minimum energy (lines 62-65). We then order all the UAVs based on their energy in ascending order (let

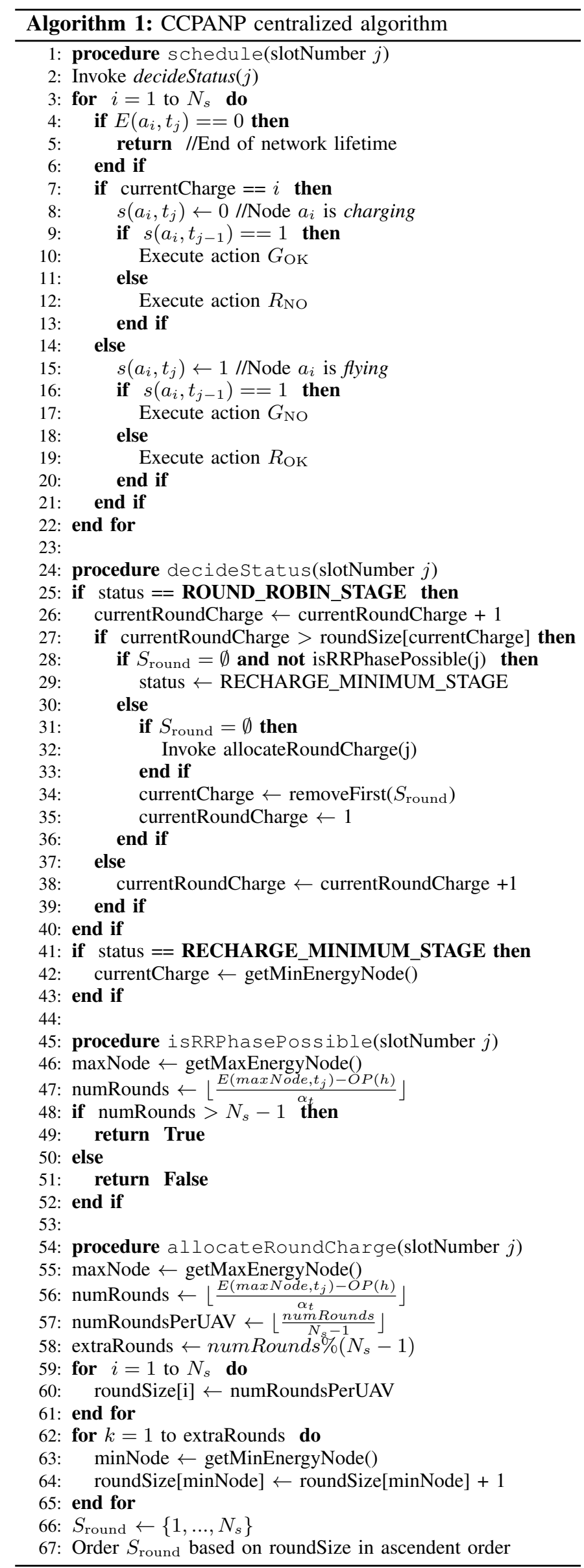


$S_{\text {round }}$ be this set at line 66-67), and in turn we extract the first element from $S_{\text {round }}$ (line 34), recharging it of roundSize $[i]$ consecutive slots (line 26-27). Once all the UAVs in $S_{\text {round }}$ have been charged once, we check whether the ROUND_ROBIN_STAGE can be iterated again through the isRRPhasePossible method (line 45-52); if so, we compute the new roundSize vector and we use a round robin fashion as explained before. Otherwise, the algorithm enters into the RECHARGE_MINIMUM_STAGE (check at line 28), where at each slot the UAV with the minimum energy is charged (line 41-43). It is easy to notice that the complexity of Algorithm 1 is bound by the allocateRoundCharge method, which is executed in $O\left(N_{s}\right)$, hence is linear with the number of UAVs. In the following, we provide numerical results about Algorithm 1.

Lemma 1 (Number of iterations). In the ROUND_ROBIN_STAGE, Algorithm 1 performs a number of iterations ${ }^{1}$ equal to:

$$
K=\log _{\psi}\left\lceil\frac{\alpha_{t} \cdot\left(N_{s}-1\right)+\frac{O P(h) \cdot \psi}{1-\psi}}{E_{\text {init }}-O P(h)+\frac{O P(h)}{1-\psi}}\right\rceil
$$

where $\psi=\frac{\beta_{t}}{\alpha_{t} \cdot\left(N_{s}-1\right)}$.

Proof. The proof is reported in the Appendix A.

Theorem 1 (Network Lifetime). The network lifetime $T$ of Algorithm 1 is in range: $\left\{T_{\mathrm{RR}} \ldots T_{\mathrm{RR}}+N_{s}-1\right\}$. We denote with $T_{\mathrm{RR}}$ and $T_{\mathrm{MIN}}$ the number of steps executed by Algorithm 1 while being in ROUND_ROBIN_STAGE and RECHARGE_MINIMUM_STAGE, respectively. Clearly, $T=T_{\mathrm{RR}}+T_{\mathrm{MIN}}$ and $0 \leq T_{\mathrm{MIN}}<N_{s}-1$.

Proof. The proof is reported in the Appendix B.

Corollary 1 (Number of swaps). The maximum number of charge swaps, i.e. of the number of changes of the UAV currently under charge, is in range $\left\{N_{s} \cdot K \ldots N_{s} \cdot K+N_{s}-1\right\}$ for the Algorithm 1 ( $K$ is the value given by (8)).

Proof. The proof is reported in the Appendix C.

We now prove the optimality of the proposed algorithm in terms of network lifetime maximization, using a two-step approach. First, we prove the optimality of Algorithm 1 when $\gamma_{h}=0$ and $\delta_{h}=0$, i.e. with no penalties for the swap operations. Then, we prove that Algorithm 1 minimizes the number of swap operations with $\gamma_{h}, \delta_{h}>0$.

Theorem 2 (Optimality1). If $\gamma_{h}=0$ and $\delta_{h}=0$, then Algorithm 1 guarantees the maximum lifetime, i.e. $t_{\text {final }}$ is maximum.

Proof. The proof is reported in the Appendix D.

Theorem 3 (Optimality2). If $\gamma_{h}>0, \delta_{h}>0$, Algorithm 1 minimizes the number of charge swaps.

Proof. The proof is reported in the Appendix E.

\footnotetext{
${ }^{1}$ An iteration of the ROUND_ROBIN_STAGE mode is completed when all the UAV nodes have been recharged. Each UAV $a_{i}$ charges for a number of slots given by roundSize $(i)$.
}

Theorem 4 (Optimality3). The Algorithm 1 is able to satisfy the $\rho_{j} \geq \kappa$ constraint for every $t_{j}<t_{\text {final }}$.

Proof. The proof is reported in the Appendix F.

\section{Distributed Game-Theory Based Approach}

Despite its optimality, Algorithm 1 is not conducive for easy implementation since it assumes strict coordination among the UAVs. We now describe an alternate distributed approach including mechanisms for the scheduling of recharging operations and for the distributed positioning of the UAVs. The first component (i.e. charge scheduling) is modeled via gametheory techniques: Section V-A introduces the formulation and the computation of the mixed strategies meeting the Nash equilibrium. Based on it, three different schedulers are proposed in Section V-B with varying levels of knowledge sharing among the UAVs. The second component of positioning the UAVs involves a distributed bio-inspired algorithm, presented in Section V-D.

Although we are aware that distributed charging scheduling problem can be addressed also via other techniques, like gossiping mechanisms [32], or distributed network leader elections [33], the choice of the game-theoretical formulation provides two main advantages: $(i)$ it guarantees convergence to a coordinated solution within a decentralized environment, hence also maximizing reliance in presence of hardware/software failures of the UAVs; (ii) it allows decoupling the strategy played by the UAVs from the information dissemination process, i.e. from the amount of knowledge available at each UAV, as better detailed in the following. Moreover, we remark that in this paper we are considering the scheduling process as a set of consecutive and different static games at each time slot $t_{j} \in T$. Our modeling aims to cope with the unpredictable and unknown dynamics of the environment: at each instant, all the UAVs will adapt their behaviours to the actual internal/external conditions, e.g. their residual energy, and take proper decisions. The proposed solutions can also deal with dynamic network scenarios in which the number of UAVs can change over time. Given the requirements to adapt the system response to varying environmental conditions, our formulation does not take into account the relationships among temporal subgames, i.e. it does not track the system temporal evolution. We plan to further elaborate on this issue as future work.

TABLE II

GENERAL GAME DESCRIPTION

\begin{tabular}{|c|c|c|c|}
\hline S & Strategy & Z & Utility functions \\
\hline \hline$s_{\mathrm{fly}}$ & $p_{G}^{i, j}$ & $\boldsymbol{G}_{\mathrm{OK}}$ & $u^{i, j}\left(G_{\mathrm{OK}}\right)$ \\
\hline$s_{\mathrm{fly}}$ & $\left(1-p_{G}^{i, j}\right)$ & $\boldsymbol{G}_{\mathrm{NO}}$ & $u^{i, j}\left(G_{\mathrm{NO}}\right)$ \\
\hline$s_{\text {rec }}$ & $p_{R}^{i, j}$ & $\boldsymbol{R}_{\mathrm{OK}}$ & $u^{i, j}\left(R_{\mathrm{OK}}\right)$ \\
\hline$s_{\text {rec }}$ & $\left(1-p_{R}^{i, j}\right)$ & $\boldsymbol{R}_{\mathrm{NO}}$ & $u^{i, j}\left(R_{\mathrm{NO}}\right)$ \\
\hline
\end{tabular}

\section{A. Game Formulation and Resolution}

Without loss of generality, we model the UAV scheduling operations according to the normal-form game defined as 
follows.

Definition 2. At each time slot $t_{j} \in T$, the normal-form game is defined as the triple $\left\langle A, Z, u^{j}\right\rangle$, where:

- $A=\left\{a_{1}, a_{2}, \ldots, a_{N_{S}}\right\}$ is the set of UAVs/players;

- $Z=\left\{G_{\mathrm{OK}}, G_{\mathrm{NO}}, R_{\mathrm{OK}}, R_{\mathrm{NO}}\right\}$ is the action set available to each player. The meaning of each action has been described in Section III-A;

- $u^{j}=\left\{u^{1, j}, u^{2, j}, \ldots, u^{N_{S}, j}\right\}$ is the profile of the utility functions at time slot $t_{j}$, where $u^{i, j}$ is the utility function or payoff function for player $a_{i}$, i.e. $u^{i, j}: Z \rightarrow \mathbb{R}$.

Let $\theta^{i, j}=\left\{p_{G}^{i, j},\left(1-p_{G}^{i, j}\right), p_{R}^{i, j},\left(1-p_{R}^{i, j}\right)\right\}$ be the strategy for player $a_{i}$ at time slot $t_{j}$ defining the probability distribution over the set of possible actions $Z$. Here, $p_{G}^{i, j},\left(1-p_{G}^{i, j}\right), p_{R}^{i, j}$ and $\left(1-p_{R}^{i, j}\right)$ denote the probabilities to execute actions $G_{\mathrm{OK}}$, $G_{\mathrm{NO}}, R_{\mathrm{OK}}$ and $R_{\mathrm{NO}}$, respectively.

We have a mixed strategy if more than one action in $Z$ is associated to a non-zero probability; the corresponding actions are called support of the mixed strategy. Let $\Theta^{i, j}$ be the set of all possible mixed strategies for player $a_{i}$ at time slot $t_{j}$. Finally, let $\Theta^{j}=\Theta^{1, j} \times \Theta^{2, j} \times \cdots \times \Theta^{N_{S}, j}$ be the set of all strategy profiles at time slot $t_{j}$.

We define $\Theta^{i, j} \doteq \Theta_{G}^{i, j}$ as the set of all possible mixed strategies for player $a_{i}$ being in state $s_{\text {fly }}$ (flying). In accordance with the state definition of Section III-A, the support of $\Theta_{G}^{i, j}$ includes $G_{\mathrm{OK}}$ and $G_{\mathrm{NO}}$ only. Moreover, we consider the mixed strategy $\theta_{G}^{i, j} \in \Theta_{G}^{i, j}$, defined as: $\theta_{G}^{i, j}=\left\{p_{G}^{i, j},(1-\right.$ $\left.\left.p_{G}^{i, j}\right), 0,0\right\}$. Similarly, we define $\Theta^{i, j} \doteq \Theta_{R}^{i, j}$ as the set of all possible mixed strategies for player $a_{i}$ being in state $s_{\text {rec }}$ (recharging). In this case, the support includes $R_{\mathrm{OK}}$ and $R_{\mathrm{NO}}$ only. Again, we consider the mixed strategy $\theta_{R}^{i, j} \in \Theta_{R}^{i, j}$, defined as: $\theta_{R}^{i, j}=\left\{0,0, p_{R}^{i, j},\left(1-p_{R}^{i, j}\right)\right\}$. Table II depicts the game description where we also indicate (as first column) the current state of UAV $a_{i} \in A$ at time slot $t_{j-1} \in T$, in order to be able to execute the action indicated in the third column. The goal of the analysis reported in the following is to compute the optimal values of $p_{G}^{i, j}$ and $p_{R}^{k, j}$, so that the system achieves a Nash-equilibrium. The final results of the analysis are constituted by Theorems 5 and 6 , which provide closed formulations of $p_{G}^{i, j}$ and $p_{R}^{k, j}$. Since the game depends on the current state of the UAV, we consider two cases separately: (i) the so-called Catch Game, that is played when the UAV $a_{i}$ is in state $s_{\mathrm{fly}}$, and the (ii) the Release Game, that is played by the UAV $a_{k}$ in state $s_{\text {rec }}$. However, we highlight that such division is for ease of disposition only, since the two stages belong to the same game, although observed from two different perspectives (i.e. from the perspective of an UAV that is in $s_{\mathrm{fly}}$ or in $s_{\mathrm{rec}}$ state); moreover, as a further proof of this concept, the solution of the two stages are mutually dependant, as better detailed in the following.

1) The Catch Game: We focus on the analysis of the game played by $a_{i}$ in state $s_{\mathrm{fly}}$, having the mixed strategy $\theta_{G}^{i, j} \in \Theta_{G}^{i, j}$. At each time slot $t_{j} \in T$, we must determine the mixed strategy $\theta_{G}^{i, j}=\left\{p_{G}^{i, j},\left(1-p_{G}^{i, j}\right), 0,0\right\}$ and hence the probability $p_{G}^{i, j}$ for UAV $a_{i}$ to execute action $G_{\text {OK }}$ (i.e. attempt to occupy the replenishment station); with probability $1-p_{G}^{i, j}$, UAV $a_{i}$ executes the action $G_{\mathrm{NO}}$ (i.e. keeps flying). In game- theory, a Nash equilibrium mixed strategy is achieved when the opponents randomize their actions in order to make the player $a_{i}$ indifferent between the possible actions [35], i.e.:

$$
u^{i, j}\left(G_{\mathrm{OK}}\right)=u^{i, j}\left(G_{\mathrm{NO}}\right)
$$

We now define the utility functions $u^{i, j}\left(G_{\mathrm{OK}}\right)$ and $u^{i, j}\left(G_{\mathrm{NO}}\right)$. It is easy to notice that the execution of action $G_{\mathrm{OK}}$ can lead to two situations: $(i)$ if $\phi\left(t_{j}\right)=1$, then the UAV $a_{i}$ moves to state $s_{\text {rec }}$, occupies the station $S_{E}$ and starts recharging its battery $\left(s\left(a_{i}, t_{j}\right)=1\right)$; (ii) if $\phi\left(t_{j}\right)=0$, then the UAV remains in state $s_{\mathrm{fly}}\left(s\left(a_{i}, t_{j}\right)=0\right)$. Let $U_{F}^{i, j}\left(G_{\mathrm{OK}}\right)$ and $U_{B}^{i, j}\left(G_{\mathrm{OK}}\right)$ be the payoffs received by UAV $a_{i}$ in the two cases mentioned before. Moreover, let $p_{B}^{i, j}$ be the probability for UAV $a_{i}$ to find the station $S_{E}$ occupied by another UAV at time slot $t_{j}$. We can then express the utility function $u^{i, j}\left(G_{\mathrm{OK}}\right)$ as follows:

$$
u^{i, j}\left(G_{\mathrm{OK}}\right)=p_{B}^{i, j} \cdot U_{B}^{i, j}\left(G_{\mathrm{OK}}\right)+\left(1-p_{B}^{i, j}\right) \cdot U_{F}^{i, j}\left(G_{\mathrm{OK}}\right)
$$

Similarly, we can define the utility function $u^{i, j}\left(G_{\mathrm{NO}}\right)$ as follows:

$$
u^{i, j}\left(G_{\mathrm{NO}}\right)=p_{B}^{i, j} \cdot U_{B}^{i, j}\left(G_{\mathrm{NO}}\right)+\left(1-p_{B}^{i, j}\right) \cdot U_{F}^{i, j}\left(G_{\mathrm{NO}}\right)
$$

The functions $U_{B}^{i, j}\left(G_{\mathrm{NO}}\right)$ and $U_{F}^{i, j}\left(G_{\mathrm{NO}}\right)$ are the payoffs that UAV $a_{i}$ receives if the station $S_{E}$ remains busy $\left(\phi\left(t_{j}\right)=0\right)$ or free $\left(\phi\left(t_{j}\right)=1\right)$ during time slot $t_{j}$, respectively. How to define the payoff functions $U_{B}^{i, j}\left(G_{\mathrm{OK}}\right), U_{B}^{i, j}\left(G_{\mathrm{NO}}\right), U_{F}^{i, j}\left(G_{\mathrm{OK}}\right)$ and $U_{F}^{i, j}\left(G_{\mathrm{NO}}\right)$ is explained in Section V-B. Substituting (10) and (11) into (9), we can get a closed formulation of $p_{B}^{i, j}$ as a function of the payoff functions $U_{B}^{i, j}(\cdot)$ and $U_{F}^{i, j}(\cdot)$, i.e.

$p_{B}^{i, j}=\frac{U_{F}^{i, j}\left(G_{\mathrm{NO}}\right)-U_{F}^{i, j}\left(G_{\mathrm{OK}}\right)}{U_{B}^{i, j}\left(G_{\mathrm{OK}}\right)-U_{F}^{i, j}\left(G_{\mathrm{OK}}\right)+U_{F}^{i, j}\left(G_{\mathrm{NO}}\right)-U_{B}^{i, j}\left(G_{\mathrm{NO}}\right)}$

Let $a_{k}$ be the UAV which is using the station $S_{E}$ at time slot $t_{j-1}$. With probability $p_{R}^{k, j}$, UAV $a_{k}$ can release the station by executing action $R_{\mathrm{OK}}$ at the beginning of time slot $t_{j}$; conversely, with probability $\left(1-p_{R}^{k, j}\right)$, UAV $a_{k}$ keeps recharging also during time slot $t_{j}$ by executing the action $R_{\mathrm{NO}}$. Now, the probability $p_{B}^{i, j}$ can be computed as the opposite of the idle case, that occurs when UAV $a_{k}$ releases the station and then no one will try to catch it during slot $t_{j}$. More formally:

$$
p_{B}^{i, j}=1-\left(p_{R}^{k, j} \cdot \prod_{a_{h} \in A \backslash\left\{a_{i}, a_{k}\right\}}\left(1-p_{G}^{h, j}\right)\right)
$$

We observe that the case where no UAV $a_{k}$ is recharging at time slot $t_{j-1}$ is a special instance of (13) with $p_{R}^{k, j}=1$. Finally, we derive $p_{G}^{i, j}$ through the following Theorem.

Theorem 5. For the Nash-equilibrium, the probability $p_{G}^{i, j}$ for $U A V a_{i}$ to choose action $G_{\mathrm{OK}}$ at the beginning of time slot $t_{j}$ must be defined as follows:

$$
p_{G}^{i, j}=1-\sqrt[\left(N_{S}-1\right)]{\frac{\prod_{a_{h} \in A \backslash\left\{a_{i}\right\}}\left(1-p_{B}^{h, j}\right)}{\left(1-p_{B}^{i, j}\right)^{N_{S}-2} \cdot p_{R}^{k, j}}}
$$

where $p_{R}^{k, j}$ is the probability defined by (22) in case $\phi\left(t_{j-1}\right)=$ 0 , it is equal to 1 otherwise. 
Proof. The proof is reported in the Appendix G.

Remark. Since $0 \leq p_{B}^{i, j} \leq 1$, we can derive from (12) the following constraints on the settings of the payoffs $U_{F}^{i, j}\left(G_{\mathrm{NO}}\right)$, $U_{F}^{i, j}\left(G_{\mathrm{OK}}\right), U_{B}^{i, j}\left(G_{\mathrm{OK}}\right)$ and $U_{B}^{i, j}\left(G_{\mathrm{OK}}\right):$

$$
\left\{\begin{array}{l}
U_{F}^{i, j}\left(G_{\mathrm{NO}}\right)>U_{F}^{i, j}\left(G_{\mathrm{OK}}\right) \\
U_{B}^{i, j}\left(G_{\mathrm{OK}}\right)>U_{B}^{i, j}\left(G_{\mathrm{NO}}\right) \\
U_{B}^{i, j}\left(G_{\mathrm{OK}}\right)-U_{F}^{i, j}\left(G_{\mathrm{OK}}\right)+U_{F}^{i, j}\left(G_{\mathrm{NO}}\right)-U_{B}^{i, j}\left(G_{\mathrm{NO}}\right)>0
\end{array}\right.
$$

or

$$
\left\{\begin{array}{l}
U_{F}^{i, j}\left(G_{\mathrm{NO}}\right)<U_{F}^{i, j}\left(G_{\mathrm{OK}}\right) \\
U_{B}^{i, j}\left(G_{\mathrm{OK}}\right)<U_{B}^{i, j}\left(G_{\mathrm{NO}}\right) \\
U_{B}^{i, j}\left(G_{\mathrm{OK}}\right)-U_{F}^{i, j}\left(G_{\mathrm{OK}}\right)+U_{F}^{i, j}\left(G_{\mathrm{NO}}\right)-U_{B}^{i, j}\left(G_{\mathrm{NO}}\right)<0
\end{array}\right.
$$

2) Release game: We now focus on the analysis of the game played by UAV $a_{k}$ in state $s_{\text {rec }}$, having the mixed strategy $\theta_{R}^{k, j} \in \Theta_{R}^{k, j}$. At each time slot $t_{j} \in T$, we must determine the mixed strategy $\theta_{R}^{k, j}=\left\{0,0, p_{R}^{k, j},\left(1-p_{R}^{k, j}\right)\right\}$ and hence the probability $p_{R}^{k, j}$ for the UAV $a_{k}$ to execute action $R_{\mathrm{OK}}$ (i.e. release the replenishment station: $s\left(a_{k}, t_{j}\right)=1$ ); with probability $1-p_{R}^{k, j}$, UAV $a_{k}$ executes the action $R_{\mathrm{NO}}$ (i.e. keeps recharging: $\left.s\left(a_{k}, t_{j}\right)=0\right)$. As for the previous analysis, a Nash equilibrium mixed strategy is achieved when the opponents make UAV $a_{k}$ indifferent on its possible choices, i.e.:

$$
u^{k, j}\left(R_{\mathrm{OK}}\right)=u^{k, j}\left(R_{\mathrm{NO}}\right)
$$

In order to compute the value of $u^{k, j}\left(R_{\mathrm{OK}}\right)$, we distinguish two situations that might occur during slot $t_{j}$ after that UAV $a_{k}$ has executed action $R_{\mathrm{OK}}$, i.e.: ( $\left.i\right)$ no UAV attempts acquiring the station, and hence $\phi\left(t_{j}\right)=0$ or $(i i)$ at least one of the other UAVs catches the station at time slot $t_{j}$, and hence $\phi\left(t_{j}\right)=$ 1. Let $U_{T_{0}}^{k, j}\left(R_{\mathrm{OK}}\right)$ and $U_{T_{0}}^{k, j}\left(R_{\mathrm{NO}}\right)$ be the payoffs that the UAV $a_{k}$ receives by executing respectively the action $R_{\mathrm{OK}}$ or $R_{\mathrm{NO}}$, and no other UAV is trying to grab the station $S_{E}$ at time slot $t_{j}$. Similarly, let $U_{T_{+}}^{k, j}\left(R_{\mathrm{OK}}\right)$ and $U_{T_{+}}^{k, j}\left(R_{\mathrm{NO}}\right)$ be the payoffs received by UAV $a_{k}$ after executing respectively the action $R_{\mathrm{OK}}$ or $R_{\mathrm{NO}}$, and in case some other UAVs will try catching the station $S_{E}$ at time slot $t_{j}$. How to define the payoff functions $U_{T_{0}}^{k, j}\left(R_{\mathrm{OK}}\right), U_{T_{0}}^{k, j}\left(R_{\mathrm{NO}}\right), U_{T_{+}}^{k, j}\left(R_{\mathrm{OK}}\right)$ and $U_{T_{+}}^{k, j}\left(R_{\mathrm{NO}}\right)$ is explained in Section V-B. We can derive the value of $u^{k, j}\left(R_{\mathrm{OK}}\right)$ as follows:

$$
u^{k, j}\left(R_{\mathrm{OK}}\right)=p_{T_{0}}^{k, j} \cdot U_{T_{0}}^{k, j}\left(R_{\mathrm{OK}}\right)+\left(1-p_{T_{0}}^{k, j}\right) \cdot U_{T_{+}}^{k, j}\left(R_{\mathrm{OK}}\right)
$$

Similarly, the term $u^{k, j}\left(R_{\mathrm{NO}}\right)$ can be expressed as follows:

$$
u^{k, j}\left(R_{\mathrm{NO}}\right)=p_{T_{0}}^{k, j} \cdot U_{T_{0}}^{k, j}\left(R_{\mathrm{NO}}\right)+\left(1-p_{T_{0}}^{k, j}\right) \cdot U_{T_{+}}^{k, j}\left(R_{\mathrm{NO}}\right)
$$

In both the Equations above, the term $p_{T_{0}}^{k, j}$ is the probability that none of the other UAVs being in state $s_{\text {fly }}$ performs the action $G_{\mathrm{OK}}$ at time slot $t_{j}$, and it is defined as follow:

$$
p_{T_{0}}^{k, j}=\prod_{a_{h} \in A \backslash\left\{a_{k}\right\}}\left(1-p_{G}^{h, j}\right)
$$

Substituting (18) and (19) into (17), we get the formulation of $p_{T_{0}}^{k, j}$ as a function of the payoff functions $U_{T_{0}}^{k, j}$ and $U_{T_{+}}^{k, j}$, i.e.:

$p_{T_{0}}^{k, j}=\frac{U_{T_{+}}^{k, j}\left(R_{\mathrm{NO}}\right)-U_{T_{+}}^{k, j}\left(R_{\mathrm{OK}}\right)}{U_{T_{0}}^{k, j}\left(R_{\mathrm{OK}}\right)-U_{T_{+}}^{k, j}\left(R_{\mathrm{OK}}\right)+U_{T_{+}}^{k, j}\left(R_{\mathrm{NO}}\right)-U_{T_{0}}^{k, j}\left(R_{\mathrm{NO}}\right)}$

We finally introduce the Theorem above, which provides the closed form Equation of $p_{R}^{k, j}$.

Theorem 6. For the Nash-equilibrium, the probability $p_{R}^{k, j}$ for $U A V a_{k}$ being in state $s_{\mathrm{rec}}$ to choose action $R_{\mathrm{OK}}$ at the beginning of time slot $t_{j}$ must be defined as follow:

$$
p_{R}^{k, j}=\sqrt[\left(N_{S}-1\right)]{\frac{\prod_{a_{h} \in A \backslash\left\{a_{k}\right\}}\left(1-p_{B}^{h, j}\right)}{\left(p_{T_{0}}^{k, j}\right)^{N_{S}-2}}}
$$

where $p_{B}^{h, j}$ is defined in (12).

Proof. The proof is reported in the Appendix H.

Remark. Since $0 \leq p_{T_{0}}^{k, j} \leq 1$ we can derive the following constraints on $U_{T_{0}}^{k, j}\left(R_{\mathrm{OK}}\right), U_{T_{0}}^{k, j}\left(R_{\mathrm{NO}}\right), U_{T_{+}}^{k, j}\left(R_{\mathrm{OK}}\right)$ and $U_{T_{+}}^{k, j}\left(R_{\mathrm{NO}}\right):$

$\left\{\begin{array}{l}U_{T_{+}}^{k, j}\left(R_{\mathrm{NO}}\right)>U_{T_{+}}^{k, j}\left(R_{\mathrm{OK}}\right) \\ U_{T_{0}}^{k, j}\left(R_{\mathrm{OK}}\right)>U_{T_{0}}^{k, j}\left(R_{\mathrm{NO}}\right) \\ U_{T_{0}}^{k, j}\left(R_{\mathrm{OK}}\right)-U_{T_{+}}^{k, j}\left(R_{\mathrm{OK}}\right)+U_{T_{+}}^{k, j}\left(R_{\mathrm{NO}}\right)-U_{T_{0}}^{k, j}\left(R_{\mathrm{NO}}\right)>0\end{array}\right.$

or

$\left\{\begin{array}{l}U_{T_{+}}^{k, j}\left(R_{\mathrm{NO}}\right)<U_{T_{+}}^{k, j}\left(R_{\mathrm{OK}}\right) \\ U_{T_{0}}^{k, j}\left(R_{\mathrm{OK}}\right)<U_{T_{0}}^{k, j}\left(R_{\mathrm{NO}}\right) \\ U_{T_{0}}^{k, j}\left(R_{\mathrm{OK}}\right)-U_{T_{+}}^{k, j}\left(R_{\mathrm{OK}}\right)+U_{T_{+}}^{k, j}\left(R_{\mathrm{NO}}\right)-U_{T_{0}}^{k, j}\left(R_{\mathrm{NO}}\right)<0\end{array}\right.$

\section{B. Scheduling Algorithms}

In this Section, we define the values of the payoffs used by the Catch and Release games previously introduced. More specifically, we consider three different formulations of the payoffs, corresponding to three different scheduling algorithms.

- Global knowledge: we assume that each UAV $a_{i}$ knows the residual energy $E\left(a_{h}, t_{j}\right)$ of all the UAVs in the network $a_{h} \in A$ and the current state $\phi\left(t_{j}\right)$ of the station $S_{E}$, for each time slot $t_{j}<t_{\text {final }}$. The information exchange is enabled via the periodic broadcast of a STRATEGY message, every $T_{\text {STRATEGY }}$ seconds and from each UAV. The STRATEGY message includes the value of $E\left(a_{i}, t_{j}\right)$ and, for the UAV $k$ being in $s_{\text {rec }}$ state, the time instant $t_{\text {START }}^{k}$ when it started recharging. Through these values, each UAV can compute the payoff values and then the probabilities for mixed strategies (i.e. (14) and (22)). Moreover, each message is re-broadcasted hop by hop, in order to reach all the UAVs of the aerial network. Reliable communication is assumed.

- Local knowledge: we assume that each UAV $a_{i}$ knows the residual energy $E\left(a_{h}, t_{j}\right)$ of all the UAVs $a_{h} \in A$ at 
one-hop distance, for each time slot $t_{j}<t_{\text {final }}$; however, it does not have information about the utilization of the station $S_{E}$. Let $N e i g h_{i} \subseteq A$ be the set of onehop neighbours of UAV $a_{i}$. As before, the information exchange is enabled via the periodic broadcast of STRATEGY messages every $T_{\text {STRATEGY }}$ seconds, but without involving multi-hop retransmissions. The lack of global scenario knowledge implies that UAV $i$ might not be able to compute $p_{G}^{i, j}$ and $p_{R}^{i, j}$ values like (14) and (22), unless introducing some approximations, which are explained later in this Section.

- Personal knowledge: we assume that each UAV $a_{i}$ knows its residual energy $E\left(a_{i}, t_{j}\right)$ only with no STRATEGY message exchanges. Again, the approximations needed for the computation of the mixed strategies are explained later in this Section.

As before, we separately consider the Catch Game and the Release Game.

TABLE III

PAYOFFS FOR THE CATCH GAME

\begin{tabular}{|c|c|c|}
\hline$U_{F}^{i, j}\left(G_{\mathrm{OK}}\right)$ & \multicolumn{2}{|c|}{$\beta_{t}-O P(h)-\tau$} \\
\hline & Global & $\left(-\alpha_{t}-O P(h)-\tau\right) \cdot\left(1+\xi_{G}^{i, j}\right)$ \\
$U_{B}^{i, j}\left(G_{\mathrm{OK}}\right)$ & Local & $\left(-\alpha_{t}-O P(h)-\tau\right) \cdot\left(1+\xi_{L}^{i, j}\right)$ \\
& Personal & $\left(-\alpha_{t}-O P(h)-\tau\right) \cdot\left(1+\xi_{P}^{i, j}\right)$ \\
\hline & Global & $\left(-\alpha_{t}+\tau\right) \cdot\left(1+\xi_{G}^{i, j}\right)$ \\
$U_{F}^{i, j}\left(G_{\mathrm{NO}}\right)$ & Local & $\left(-\alpha_{t}+\tau\right) \cdot\left(1+\xi_{L}^{i, j}\right)$ \\
& Personal & $\left(-\alpha_{t}+\tau\right) \cdot\left(1+\xi_{P}^{i, j}\right)$ \\
\hline$U_{B}^{i, j}\left(G_{\mathrm{NO}}\right)$ & \multicolumn{2}{|c}{$-\alpha_{t}+\tau$} \\
\hline
\end{tabular}

Table III shows, for all the three algorithms defined above, the payoffs that each UAV $a_{i}$ being in state $s_{\text {fly }}$ at time slot $t_{j}$ will receive depending on the executed action $\left(G_{\mathrm{OK}}\right.$ or $\left.G_{\mathrm{NO}}\right)$ and on the $S_{E}$ state $\left(\phi\left(t_{j}\right)=0\right.$ or $\left.\phi\left(t_{j}\right)=1\right)$. Here, $\alpha_{t}, \beta_{t}$ and $O P(h)=\left(\gamma_{h}+\delta_{h}\right)$ are the system parameters introduced in Section III-A, $\tau$ is a constant parameter modeling the coverage penalty/profit, while $\xi_{G}^{i, j}, \xi_{L}^{i, j}$ and $\xi_{P}^{i, j}$ represent the energy factor of $a_{i}$ with respect to the actual knowledge, for the global, local and personal algorithms, respectively. The $\xi^{i, j}$ factors are computed as follows:

$$
\begin{gathered}
\xi_{G}^{i, j}=\frac{\underset{1 \leq h \leq N_{S}}{\operatorname{argmax}}\left(E\left(a_{h}, t_{j}\right)\right)-E\left(a_{i}, t_{j}\right)}{\underset{1 \leq h \leq N_{S}}{\operatorname{argmax}\left(E\left(a_{h}, t_{j}\right)\right)-\underset{1 \leq h \leq N_{S}}{\operatorname{argmin}}\left(E\left(a_{h}, t_{j}\right)\right)}} \\
\xi_{L}^{i, j}=\frac{\underset{h \in N \operatorname{Neigh}_{i} \cup\left\{a_{i}\right\}}{\operatorname{argmax}}\left(E\left(a_{h}, t_{j}\right)\right)-E\left(a_{i}, t_{j}\right)}{\operatorname{argmax}_{h \in N e i g h_{i} \cup\left\{a_{i}\right\}}\left(E\left(a_{h}, t_{j}\right)\right)-\underset{h \in N e i g h_{i} \cup\left\{a_{i}\right\}}{\operatorname{argmin}}\left(E\left(a_{h}, t_{j}\right)\right)} \\
\xi_{P}^{i, j}=\operatorname{MAX}\left(0, \frac{E_{\text {init }}-E_{a_{i}}}{E_{\text {init }}}\right)
\end{gathered}
$$

We notice that the values described in Table III satisfy the constraints defined in (16).

The rationale behind the values of Table III is the following: when executing the action $G_{\mathrm{OK}}$, and the station $S_{E}$ is free (i.e. the case of $\left.U_{F}^{i, j}\left(G_{\mathrm{OK}}\right)\right)$, the payoff is the energy recharged $\left(\beta_{t}\right)$ minus the energy lost for landing and flying back again $(O P(h))$. If, instead, the station $S_{E}$ is found busy (i.e. the case of $\left.U_{B}^{i, j}\left(G_{\mathrm{OK}}\right)\right)$, the payoff is always a penalty, and includes also the energy lost for remaining in flying state $\left(\alpha_{t}\right)$ : moreover, the penalty increases proportionally with $\xi^{i, j}$, i.e. based on the amount of residual energy of UAV $a_{i}$ (for the personal algorithm) or to the energy level of $a_{i}$ compared to other known players (for the global and local algorithms). Similarly, the action $G_{\mathrm{NO}}$ while the station $S_{E}$ is free (i.e. the case of $U_{F}^{i, j}\left(G_{\mathrm{NO}}\right)$ ) always leads to a penalty, which is proportional to $\alpha_{t}$ and to the residual energy of the UAV, as discussed before. Finally, the payoff of executing action $G_{\mathrm{NO}}$ with the station $S_{E}$ busy is always equal to the energy lost being in $s_{\mathrm{fly}}$ state, i.e. $\alpha_{t}$. It is easy to notice that all the above Equations contain the $\tau$ parameter, which takes into account the impact of the action been performed by UAV $a_{i}$ on the scenario coverage. When executing the action $G_{\mathrm{OK}}$, we always add a coverage penalty equal to $-\tau$, since the UAV $a_{i}$ is attempting to move on the ground, hence potentially creating a coverage hole. Vice versa, when executing the action $G_{\mathrm{NO}}$, we add a coverage profit equal to $+\tau$.

TABLE IV

PAYOFFS FOR THE RELEASE GAME

\begin{tabular}{|c|c|c|}
\hline$U_{T_{+}}^{k, j}\left(R_{\mathrm{OK}}\right)$ & \multicolumn{2}{|c|}{1} \\
\hline & Global & $-N_{S} \cdot \chi_{G}^{k, j}$ \\
$U_{T_{0}}^{k, j}\left(R_{\mathrm{OK}}\right)$ & Local & $-N_{S} \cdot \chi_{L}^{k, j}$ \\
& Personal & $-N_{S} \cdot \chi_{P}^{k, j}$ \\
\hline & Global & $-\chi_{G}^{k, j}$ \\
$U_{T_{+}}^{k, j}\left(R_{\mathrm{NO}}\right)$ & Local & $-\chi_{L}^{k, j}$ \\
& Personal & $-\chi_{P}^{k, j}$ \\
\hline$U_{T_{0}}^{k, j}\left(R_{\mathrm{NO}}\right)$ & \multicolumn{2}{|c|}{0} \\
\hline
\end{tabular}

Table IV shows, for all the three algorithms defined above, the payoffs that the UAV $a_{k}$ being in state $s_{\text {rec }}$ at time slot $t_{j}$ will receive depending on the executed action $\left(R_{\mathrm{OK}}\right.$ or $\left.R_{\mathrm{NO}}\right)$ and on the behaviour of the others UAVs. The rationale behind the values is the following: when executing the action $R_{\mathrm{OK}}$, and at least one other UAV attempts to occupy the station $S_{E}$ (i.e. the case of $U_{T_{+}}^{k, j}\left(R_{\mathrm{OK}}\right)$ ), we give a unit payoff since the station $S_{E}$ will not remain idle for the time slot $t_{j}$. Instead, when executing action $R_{\mathrm{OK}}$, and no other UAV is willing to occupy the station $S_{E}$ (i.e. the case of $U_{T_{+}}^{k, j}\left(R_{\mathrm{NO}}\right)$ ), we apply a penalty proportional to the number of UAVs $N_{S}$ and to the estimated optimal recharge time. To this aim, let $\chi_{G}^{k, j}, \chi_{L}^{k, j}$ and $\chi_{P}^{k, j}$ be the ratio between the time spent by player $a_{k}$ into the recharging station $S_{E}$ at time slot $t_{j}$, and the optimal recharge time for the global, local and personal schedulers, respectively. We compute such values as follows:

$$
\begin{gathered}
\chi_{G}^{k, j}=\operatorname{MIN}\left\{1, \frac{t_{S_{E}}^{k, j}}{t_{\mathrm{EST}}^{j}(A)}\right\} \\
\chi_{L}^{k, j}=\operatorname{MIN}\left\{1, \frac{t_{S_{E}}^{k, j}}{t_{\mathrm{EST}}^{j}\left(N e i g h_{k} \cup\left\{a_{k}\right\}\right)}\right\}
\end{gathered}
$$




$$
\chi_{P}^{k, j}=\operatorname{MIN}\left\{1, \frac{t_{S_{E}}^{k, j}}{t_{\mathrm{EST}}^{j}\left(\left\{a_{k}\right\}\right)}\right\}
$$

In the Equations above, $t_{S_{E}}^{i, j}$ is the time spent by UAV $a_{k}$ at the station $S_{E}$ till time slot $t_{j}$ and can be expressed as: $t_{S_{E}}^{i, j}=t_{j}-t_{\mathrm{START}}^{k}$, while $t_{\mathrm{EST}}^{j}(\Phi)$ is a the estimated optimal recharge time, computed similarly to Algorithm 1 (line 57), i.e.:

$$
t_{E S T}^{j}(\Phi)=\frac{\max _{E}^{j}(\Phi)-O P(h)}{\left(N_{S}-1\right) \cdot \alpha_{t}}
$$

where $\max _{E}^{j}(\Phi)$ is the maximum energy level among the UAVs $a_{h} \in \Phi$, at time slot $t_{j}$.

In the same way, we penalize UAV $a_{k}$ when executing action $R_{\mathrm{NO}}$ and at least one of the other $N_{S}-1 \mathrm{UAVs}$ is attempting to recharge its battery at the same slot $t_{j}$ (i.e. the case of $\left.U_{T_{+}}^{k, j}\left(R_{\mathrm{NO}}\right)\right)$; again, the penalty is proportional to the $\chi^{k, j}$ ratio defined before. Finally, if none of the other UAVs in state $s_{\text {fly }}$ attempt to recharge at slot $t_{j}$, we give a neutral payoff equal to 0 . We see that the values described in Table IV satisfy the constraints defined in (23).

Moreover, we remark that the Tables III and IV describe the different strategies that each UAV $a_{i} \in A$ will adopt at time slot $t_{j} \in T$ (see (14)-(22) and (12)-(21)).

\section{Approximations for the Local and Personal schedulers.}

Both (14) and (22) require global exchange of energy values among the UAVs, as well as the knowledge of $t_{\text {START }}^{k}$ for the UAV in state $s_{\mathrm{fly}}$. To address these issues, we relax the formulation of $p_{G}^{i, j}$ and $p_{R}^{i, j}$ for the Local and Personal schedulers.

- Local knowledge. In this case, the UAV $a_{i}$ gathers only the energy information from the UAV $a_{h} \in N e i g h_{i}$, i.e. in its 1-hop neighborhood. Hence, we approximate the value of $p_{G}^{i, j}$ and $p_{R}^{i, j}$ as follows:

$$
\begin{gathered}
p_{G}^{i, j}=1-\sqrt[\left(N_{S}-1\right)]{\frac{\left(\prod_{a_{h} \in N_{\text {eigh }}}\left(1-p_{B}^{h, j}\right)\right)}{\left(1-p_{B}^{i, j}\right)^{N_{S}-2} \cdot \bar{p}_{R}^{i, j}}} . \\
p_{R}^{i, j}=\sqrt[\left(N_{S}-1\right)]{\frac{\left(1-\bar{p}_{B}^{i, j}\right)^{\left(N_{S}-1-\left|N e i g h_{i}\right|\right)}}{\frac{\left(\prod_{a_{h} \in N e i g h_{i}}\left(1-p_{B}^{h, j}\right)\right)}{\left(p_{T_{0}}^{i, j}\right)^{N_{S}-2}}}} \\
\cdot \sqrt[\left(N_{S}-1\right)]{\left(1-\bar{p}_{B}^{i, j}\right)^{\left(N_{S}-1-\left|N e i g h_{i}\right|\right)}}
\end{gathered}
$$

where $\bar{p}_{B}^{i, j}$ is the average of the $p_{B}^{h, j}$ with $a_{h} \in N e i g h_{i}$ and $\bar{p}_{R}^{i, j}$ is the estimation of $p_{R}^{k, j}$ for a potential UAV $a_{k}$ being in state $s_{\text {rec }}$. The value of $\bar{p}_{R}^{i, j}$ is calculated in (33) by approximating the value of $t_{S_{E}}^{k, j}$ in (29), i.e. the charging time duration for UAV $k$ that is currently using the station $S_{E}$, with the duration of the last charging operation performed by the current UAV $a_{i}$.

- Personal knowledge. In this case each UAV $a_{i}$ knows only its own residual energy $E\left(a_{i}, t_{j}\right)$, for $j \geq 0$. For this reason we greatly simplify the formulation of $p_{G}^{i, j}$ and $p_{R}^{i, j}$ by assuming constant values of $p_{B}^{h, j}=p_{B}^{i, j}$, for $1 \leq h \leq N_{S}$. Hence (14) and (22) become:

$$
\begin{gathered}
p_{G}^{i, j}=1-\sqrt[\left(N_{S}-1\right)]{\frac{\left(1-p_{B}^{i, j}\right)}{\bar{p}_{R}^{i, j}}} \\
p_{R}^{i, j}=\frac{1-p_{B}^{i, j}}{\sqrt[\left(N_{S}-1\right)]{\left(p_{T_{0}}^{i, j}\right)^{N_{S}-2}}}
\end{gathered}
$$

where $\bar{p}_{R}^{i, j}$ is defined as before for the local knowledge case.

\section{Complexity analysis}

We investigate the complexity of proposed solutions by considering both the computational complexity and the information dissemination process overhead. It is easy to notice that the computational complexity is dominated by the calculus at each time slot $t_{j}$, for each UAV $a_{i}$, of the probability $p_{G}^{i, j}$ (if $a_{i}$ is in state $s_{\mathrm{fly}}$ ) and of the probability $p_{R}^{i, j}$ (if $a_{i}$ is in state $s_{\text {rec }}$ ). Again, we treat separately the three information dissemination schemes, i,e. the global, local and personal cases.

- Global: we can notice that both $p_{R}^{i, j}$ and $p_{G}^{i, j}$ are performed in $O(N)$, since they are both characterized by the products of a sequence of $N$ terms, i.e. the $p_{B}^{*, j}$. Even if these terms depend on the computation of the $\min / \max$ variables among the $N$ UAVs (see (12) and (25)), we can assume that that these values are pre-computed before evaluating $p_{R}^{i, j}$ and $p_{G}^{i, j}$.

- Local: this case is similar to the global one, but the calculus is limited to the 1-hop neighbourhood. Here, we can consider the cardinality of $\left|N e i g h_{i}\right|, \forall a_{i} \in A$, as constant (see the next Section V-D). Hence, we can state that the computational complexity is $O(1)$.

- Personal: in this case it is easy to see that both $p_{R}^{i, j}$ and $p_{G}^{i, j}$ are $O(1)$.

To analyse the information dissemination procedure we need to examine the number of STRATEGY messages that are sent inside the UAV network. Again, we treat separately the three information dissemination schemes:

- Global: in order to implement network-wide energy information dissemination, each UAV $a_{i} \in A$ must retransmit the STRATEGY message to any other $a_{j} \in A$. Hence the number of transmitted message is $N^{2}$.

- Local: in this case there is no retransmission of messages, hence the number of transmitted message is $N$.

- Personal: here no message is transmitted at all, so the number of transmitted message is 0 .

\section{UAV Positioning}

We assume that each UAV is equipped with GPS and Wi-Fi modules, so that it can know its position, and communicate with other peers using the ad-hoc mode. Every $T_{\mathrm{BEACON}} \leq t_{\text {slot }}$ intervals, each UAV $i$ broadcasts a BEACON message containing its identifier and its position $\left(\overrightarrow{x_{i}}\right)$. The 


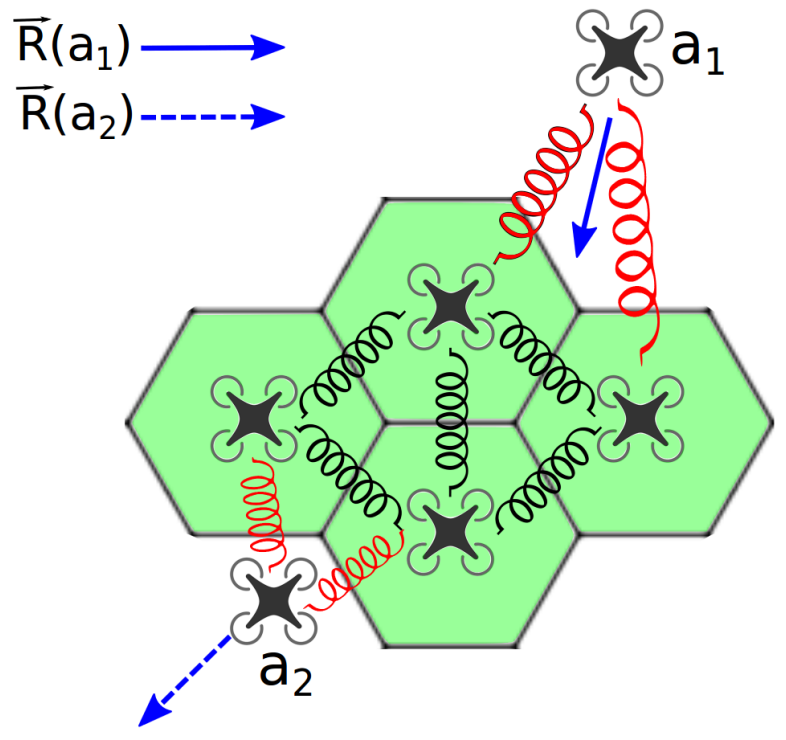

Fig. 3. The aerial mesh positioning algorithm: the virtual spring method places the UAVs according to an hexagonal pattern. The UAVs with a spring length greater then the spring equilibrium are subject to an attractive force (UAV $a_{1}$ in the Figure), while the UAVs with spring length lower then the spring equilibrium are subject to a repulsive force (UAV $a_{2}$ in the Figure)

UAV positioning algorithm extends the virtual spring model described in [9][11]. A virtual spring force $\vec{F}\left(a_{i}, a_{h}\right)$ acts between each couple of UAVs $\left(a_{i}, a_{h}\right)$ that are located at 1-hop distance, i.e. that are able to exchange the BEACON messages. The intensity of $\vec{F}\left(a_{i}, a_{h}\right)$ is computed by UAVs $a_{i}$ and $a_{h}$ according to the Hooke's law:

$$
\left|\vec{F}\left(a_{i}, a_{h}\right)\right|=-\left(\left|\overrightarrow{x_{i}}-\overrightarrow{x_{h}}\right|-d_{\mathrm{EQ}}\right) \cdot k_{\mathrm{ST}}
$$

Here, the first term is the spring displacement, given by the difference between the current distance from UAV $a_{i}$ to UAV $a_{h}$ and the length in equilibrium of the spring, indicated by $d_{\mathrm{EQ}}$. In our case, $d_{\mathrm{EQ}}$ is equal to $R \cdot \sqrt{3}$ (here $R$ is the radius of $\operatorname{Cov}(h)$ in (1)), which is the distance among the UAVs when they are positioned according to hexagonal patterns for the optimal scenario coverage [34]. The force is attractive when the distance between the UAVs is greater than $d_{\mathrm{EQ}}$, repulsive otherwise. The term $k_{\mathrm{ST}}$ is the stiffness of the spring, and is assumed to be a constant value. Every $T_{\mathrm{BEACON}}$ seconds, each UAV $a_{i}$ gathers the BEACON messages from its 1-hop neighbours $\left(N e i g h_{i}\right)$. Then, it determines $\vec{F}\left(a_{i}, a_{h}\right)$ for each neighbour $h \in N e i g h_{i}$, and it computes the resultant force $\vec{R}\left(a_{i}\right)=\sum_{h \in \text { Neigh }_{i}} \vec{F}\left(a_{i}, a_{h}\right)$. If the module of $\vec{R}\left(a_{i}\right)$ is greater than a threshold value that is analogous to inertia of a mechanical system, then $U A V a_{i}$ moves towards the direction of the resultant force in a fixed step (see Figure 3). In this way the proposed method balances the 'push' and 'pull' forces, and avoids oscillations in the ensuing movements.

\section{Vi. Performance Evaluation}

In this Section, we evaluate the proposed CCPANP problem solutions via a simulation study in a $3 \mathrm{D}$ network scenario in OMNeT++ [36]. We design and implement a comprehensive set of simulation models of UAV mobility, battery usage, and wireless communication protocols. We compared eight different algorithms, corresponding to four main approaches:

- a centralized optimal solution based on Algorithm 1 (denoted as Algo 1 in the following), assuming global coordination and complete scenario knowledge. This algorithm provides an upper bound to the system performance;

- a no-recharge solution where the recharge station $S_{E}$ is not present on the ground, and hence the UAVs must stay in the $s_{f l y}$ state all the time. We indicate with Norec such solution, which provides a lower bound to the system performance;

- the three game-theory based distributed algorithms described in Section V-B. More in details, we consider three variants based on the amount of information exchanged by the UAVs:

- a global cooperation algorithm $\left(\right.$ Game $\left._{G}\right)$ implementing the global knowledge game of Section V-B;

- a local cooperation algorithm $\left(\right.$ Game $\left._{L}\right)$ implementing the local knowledge game of Section V-B;

- a personal cooperation algorithm $\left(\right.$ Game $\left._{L}\right)$ implementing the personal knowledge game of Section V-B.

All the variants use the virtual spring algorithm of Section V-D for the distributed UAV positioning.

- three distributed probabilistic schemes which let each UAV $i$ recharge at slot $j$ with probability $P R_{i, j}$. Again, we consider three variants of the $P R_{i, j}$ function, based on the amount of information exchanged by the UAVs:

- a global cooperation probabilistic algorithm $\left(\operatorname{Prob}_{G}\right)$ : the $P R_{i, j}$ value is computed by UAV $i$ at slot $j$ by comparing its actual energy level $E\left(a_{i}, t_{j}\right)$ with those of the most charged and discharged UAVs in the network:

$$
P R_{i, j}=\frac{\underset{1 \leq h \leq N_{S}}{\operatorname{argmax}} E\left(a_{h}, t_{j}\right)-E\left(a_{i}, t_{j}\right)}{\underset{1 \leq h \leq N_{S}}{\operatorname{argmax}} E\left(a_{h}, t_{j}\right)-\underset{1 \leq h \leq N_{S}}{\operatorname{argmin}} E\left(a_{h}, t_{j}\right)}
$$

- a local knowledge probabilistic algorithm $\left(\operatorname{Prob}_{L}\right)$ : the $P R_{i, j}$ value is computed by $\mathrm{UAV} i$ at slot $j$ by comparing its actual energy level $E\left(a_{i}, t_{j}\right)$ with those of the most charged and discharged UAVs in its 1-hop neighborhood, and is defined as follows:

$$
P R_{i, j}=\frac{\underset{h \in \text { Neigh }_{i}^{+}}{\operatorname{argmax}} E\left(a_{h}, t_{j}\right)-E\left(a_{i}, t_{j}\right)}{\underset{h \in \text { Neigh }_{i}^{+}}{\operatorname{argmax}} E\left(a_{h}, t_{j}\right)-\underset{h \in \operatorname{Neigh}_{i}^{+}}{\operatorname{argmin}} E\left(a_{h}, t_{j}\right)}
$$

where $\mathrm{Neigh}_{i}^{+}=N e i g h_{i} \cup\left\{a_{i}\right\}$.

- a personal knowledge probabilistic algorithm $\left(\operatorname{Prob}_{P}\right)$ : the $P R_{i, j}$ value is computed by UAV $i$ at slot $j$ by comparing its actual energy level $E\left(a_{i}, t_{j}\right)$ with the initial battery capacity $E_{\text {init }}$, i.e. $P R_{i, j}=\frac{E\left(a_{i}, t_{j}\right)}{E_{\text {init }}}$.

For all the three probabilistic schemes described above, we consider a fixed charging time duration equal to Prob $_{\text {rec }}$ time slots. The UAV positioning is handled by the virtual spring algorithm. 


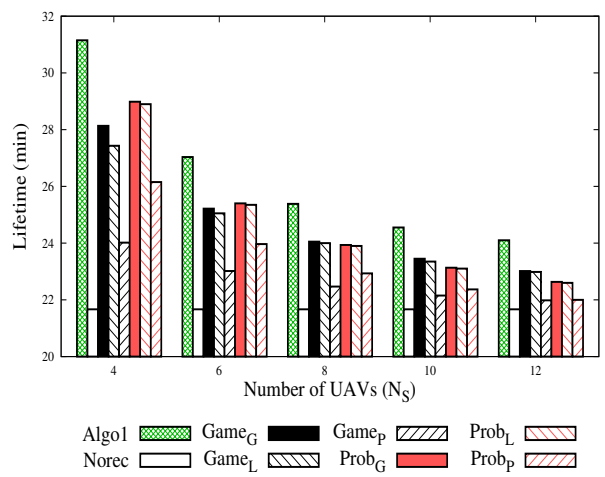

(a)

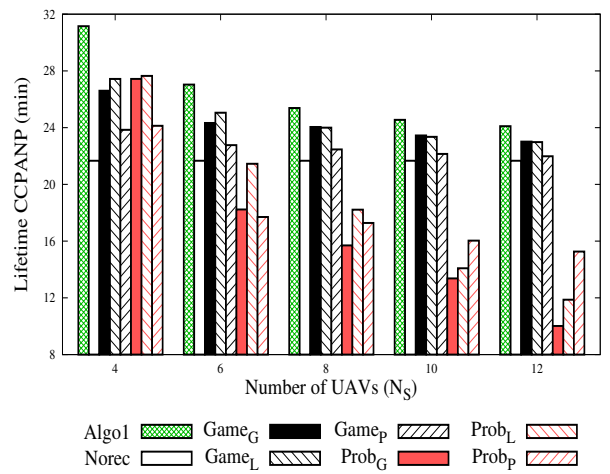

(b)

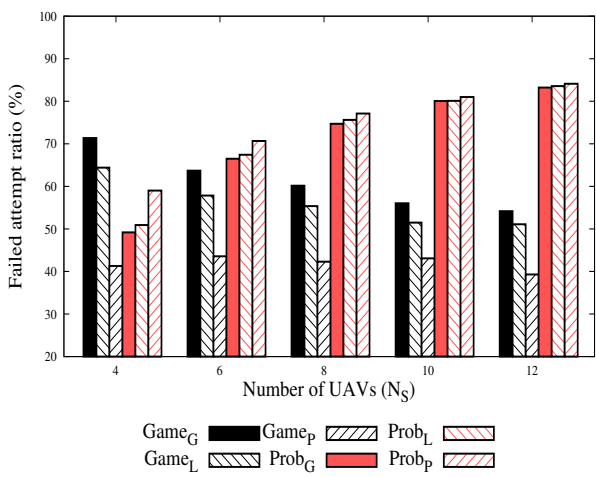

(c)

Fig. 4. The performance indexes as a function of $N_{S}$. The lifetime is shown in Figure 4(a); the CCPANP lifetime is shown in Figure 4(b); the Failed attempt ratio is shown in Figure 4(c).

The performance evaluation focuses on three quality indexes:

- Lifetime $\left(L_{\mathrm{final}}\right)$ : this index is a measure of the system lifetime, computed as the $t_{\text {final }}$ value introduced in Section III-A, i.e. the time slot in which the first UAV runs out of battery. We consider also an alternative metric for lifetime, which also accommodates the coverage and service persistence constraints of the CCPANP problem.

- CCPANP lifetime $\left(L_{\mathrm{CCPANP}}\right)$ : this index is an alternate measure of the system lifetime, measured as smallest time slot $t_{j} \in T$ such that at least one of the constrains of the CCPANP problem in the Definition 1 is not satisfied, i.e.: (i) one UAV runs out of battery or (ii) the current mesh coverage at slot $t_{j}$, i.e. $C\left(t_{j}\right)$, becomes lower than the $\kappa$ threshold for a given number of consecutive seconds. We introduce the parameter $\Delta t_{\kappa}$ which defines the maximum time interval in which the $\kappa$ constraint can be violated, thus allowing small interruptions of the coverage service.

- Failed attempt ratio $\left(F_{\text {ratio }}\right)$ : this index defines the ratio between the failed recharge attempts and the total recharge attempts performed by the UAVs.

Unless stated otherwise, we used the following setting of the system parameters: $N_{S}=8, T_{\mathrm{BEACON}}=1 s, T_{\mathrm{STRATEGY}}=$ $1 s, \theta=\frac{2}{3} \cdot \pi, \kappa=0.75, \Delta t_{\kappa}=10 s, h=20 \mathrm{~m}, k_{\mathrm{ST}}=1$, Prob $_{\text {rec }}=1, \tau=150, E_{\text {init }}=130 \mathrm{~kJ}, \alpha=100 \mathrm{~W}$, $\beta=25 W, \gamma=5 \mathrm{~J}, \delta=5 \mathrm{~J}$ (we modeled an UAV equipped with a generic 3-cell ( $3 S$ ) LiPo $11.1 \mathrm{~V}$ battery with $3250 \mathrm{mAh}$ with an approximated flight autonomy of 20 minutes and a full recharge time of 80 minutes).

We split the performance analysis in three parts. Section VI-A investigates the relationship between system performance and scenario deployment characteristics, like the number of UAVs and the altitude from the ground. Section VI-B shows the impact of system parameters related to the recharge/discharge operations. Finally, Section VI-C investigates how cooperation parameters, like the frequency of the STRATEGY messages exchanged among the UAVs, affect the system performance.

\section{A. Scenario analysis}

In this Section, we analyze the performance of the algorithms by varying characteristics like the number of available UAVs $\left(N_{S}\right)$ and the flight altitude $(h)$. Figure 4(a) shows the $L_{\text {final }}$ metric on the $y$ axis, when varying the $N_{S}$ value on the $x$ axis. We adopted the following order in this figure, and in the following histograms: the first bar is the Algol method; the second bar represents the Norec scheme; the next three bars depict the distributed game-theory approaches considering the three variants based on the amount of cooperation among the UAVs, i.e. the Game $_{G}$, Game $_{L}$ and Game $_{P}$ schemes; finally, the last three bars correspond to the probabilistic approaches considering again the three variants according to the amount of cooperation, i.e.: $\operatorname{Prob}_{G}, \operatorname{Prob}_{L}$ and $\operatorname{Prob}_{P}$. We notice that: (i) the Norec scheme performs worst, as expected; (ii) while the global cooperation schemes $\left(\right.$ Game $_{G}$ and $\left.\operatorname{Prob}_{G}\right)$ always outperform the personal solutions (i.e. Game $P$ and $\operatorname{Prob}_{P}$ ), they do not provide significant gains over local cooperation schemes $\left(\right.$ Game $_{L}$ and $\operatorname{Prob}_{L}$ ); (iii) the game-theory based schemes perform worse than the probabilistic ones with few UAVs (i.e. $N_{S} \leq 4$ ), while the trend reverses for $N_{S} \geq 6$. The results in Figure 4(a) only take into account the energy issue, but do not consider the coverage constraint $\kappa$. Figure 4(b) depicts the $L_{\text {CCPANP }}$ metric. From $N_{S}>4$, all the gametheory based schemes perform better then the probabilistic ones. Also, it is interesting to notice that the Game $_{L}$ scheme provides almost the same performance than Game $_{G}$, and pretty close to Algo1, i.e. to the optimal upper bound. In other words, the distributed mobility and charging scheduler solution provides a good approximation of the optimal one, but without requiring a global controller, and introducing a much lower network overhead than Game $_{G}$. On the opposite, the probabilistic schemes do not cope well with the coverage requirements (i.e. $\kappa$ ); for $N_{S} \geq 6$, they perform even worse than the Norec method, basically nullifying the gain of the recharging operations. This behavior can be explained by considering the $F_{\text {ratio }}$ metric in Figure 4(c). Probabilistic schemes result in greater number of recharge attempts than the game-theory based schemes, and most of them fail because the charging station $S_{E}$ is found busy. The failures also increase 


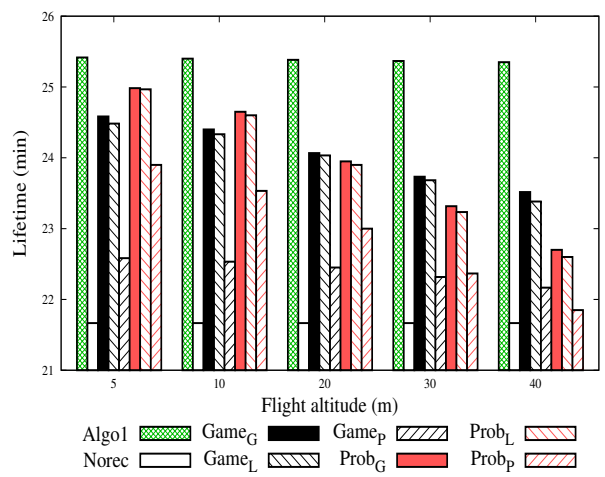

(a)

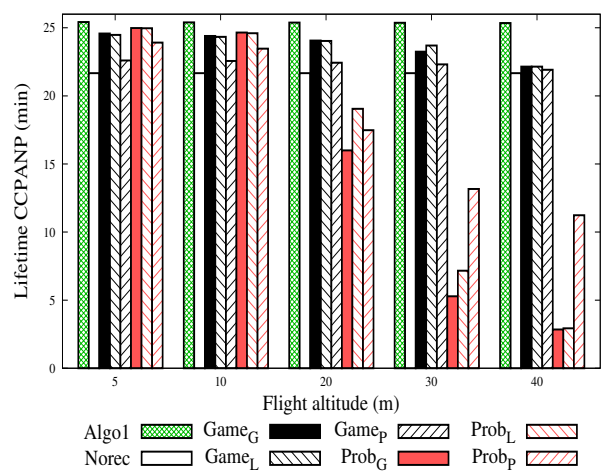

(b)

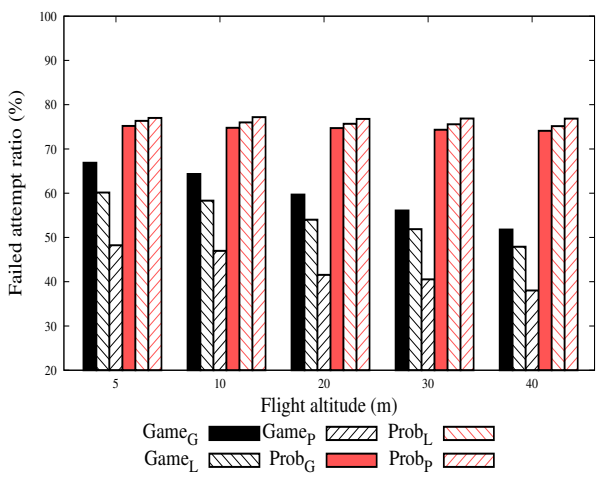

(c)

Fig. 5. The performance indexes as a function of the flight altitude $h$. The lifetime is shown in Figure 5(a); the CCPANP lifetime is shown in Figure 5(b); the Failed attempt ratio is shown in Figure 5(c).

with the number of UAVs. When the UAVs move from the air to the ground, a coverage hole may occur, driving the full coverage metric below the $\kappa$ threshold. This results in the poor performance of the probabilistic schemes in terms of $L_{\text {CCPANP }}$ metric. The game-theoretical algorithms leverage the computation of the mixed strategies, and hence, optimize the number of recharge attempts to improve the $F_{\text {ratio }}$ quality index. This trend is confirmed in Figure 6, showing the scenario coverage ratio $\rho_{j}$ (see (5)) over simulation time, for $N_{S}=8$. The spikes in the graph correspond to coverage holes, caused by single or multiple recharge attempts, and by the consequent repositioning of the UAVs according to the virtual spring mobility model. The line interruptions correspond to the $L_{\text {CCPANP }}$ lifetime values in Figure $4($ b), i.e. the time slot $t_{j}$ after which the energy or the coverage $(\kappa)$ constraints are no more satisfied. We notice that on average, the gametheory based approaches remain above the value of $\rho>0.9$. Conversely, the probabilistic methods present large decrease in coverage due the number of recharge attempts at each slot. Also, the spikes increase in frequency when the average UAV battery power level decreases. Such spikes tend to violate the $\kappa$ constraint for more than consecutive $\Delta t_{\kappa}$ seconds. Hence, the Game $_{L}$ scheme provides a performance gain of around $+30 \% L_{\text {CCPanp }}$ lifetime compared to the $\operatorname{Prob}_{L}$ scheme.

The next analysis focuses on the impact of the flight altitude (i.e. $h$ ) on the system performance. Figure 5(a) depicts the $L_{\text {final }}$ index when varying the flight altitude from $h=5 \mathrm{~m}$ to $h=40 \mathrm{~m}$. We recall that the variable $h$ impacts on the energy cost of ascending/descending operations (the $\gamma_{h}$ and $\delta_{h}$ parameters of Section III) and on the UAV coverage radius $\left(R=h \cdot \tan \left(\frac{\theta}{2}\right)\right)$. The trend of the $L_{\text {final }}$ index is similar to the previous analysis shown in Figure 4(a), i.e. the probabilistic approaches initially outperform the game theoretical methods, but provide much lower lifetime when $h \geq 20 \mathrm{~m}$, i.e. when the energy costs of ascending/descending operations become significative. Figure 5(b) shows the $L_{\mathrm{CCPANP}}$ metric over $h$. In all the distributed schemes, the UAVs need to fly over a longer distance after each recharge attempt, as the separation distance between the UAVs is a function of the sensing radius $R$, and thus of $h$. Hence, the probability of not meeting the

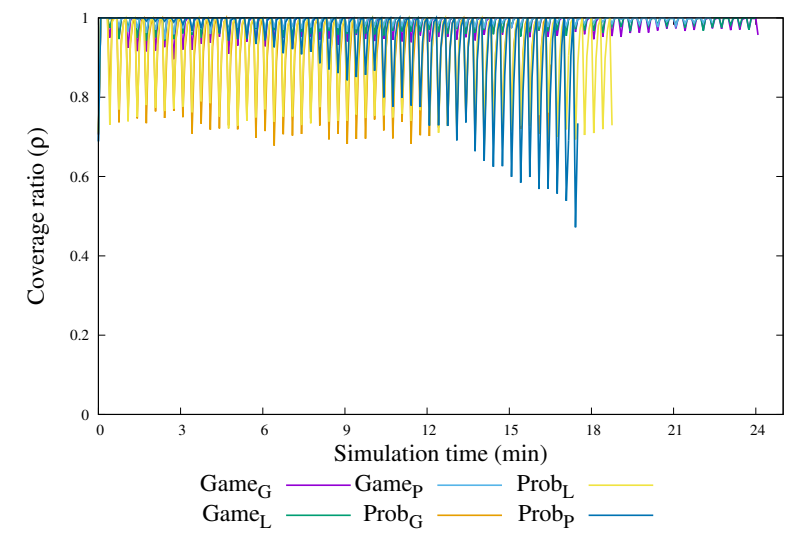

Fig. 6. The value of $\rho_{j}$ over the simulation time.

minimum coverage ratio $\kappa$ increases with the flight altitude. However, the game-theory based approaches still outperform the probabilistic ones, and the gain becomes more relevant when increasing the altitude. This is confirmed by Figure 5(c) showing the $F_{\text {ratio }}$ metric over $h$, and demonstrating that the probability schemes $\left(\operatorname{Prob}_{G}, \operatorname{Prob}_{L}\right.$ and $\left.\operatorname{Prob}_{P}\right)$ perform an excessive number of recharge attempts. Vice versa, the Game $_{G}$, Game $_{L}$ and Game $_{P}$ schemes are able to cope with the increasing altitude owing to better scheduling of the recharge operations.

\section{B. Parameters analysis}

In this Section, we explore the impact of the UAV parameters that directly characterize the discharge/recharge operations, i.e $\alpha, \beta, \gamma$ and $\delta$, on the system performance. In the following, for ease of disposition we always set the the parameters $\gamma$ and $\delta$ to the same values, i.e. $\gamma=\delta$. In Figures 7(a), 7(b) and 7(c) we depict the $L_{\text {final }}, L_{\text {CCPANP }}$ and $F_{\text {ratio }}$ indexes, with $h=20 \mathrm{~m}$ and $N_{S}=8$. The $L_{\mathrm{CCPANP}}$ metric of the distributed game-theory based algorithms are barely affected by the above parameters; system performances decrease slowly even with an high value of $\gamma=\delta=10 \mathrm{~J}$. Vice versa, for any configuration of $\gamma$ and $\delta$, the probabilistic methods 


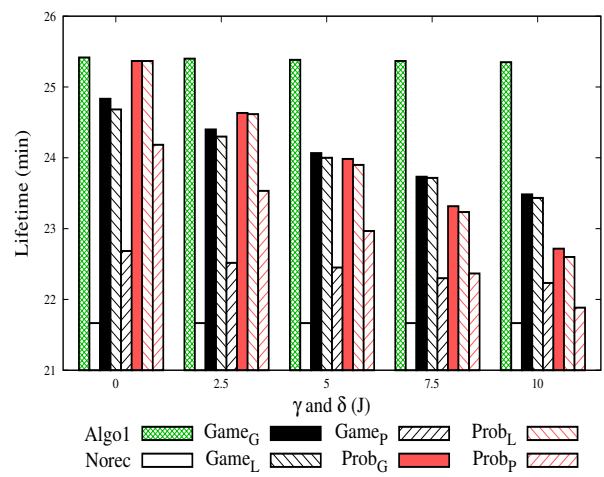

(a)

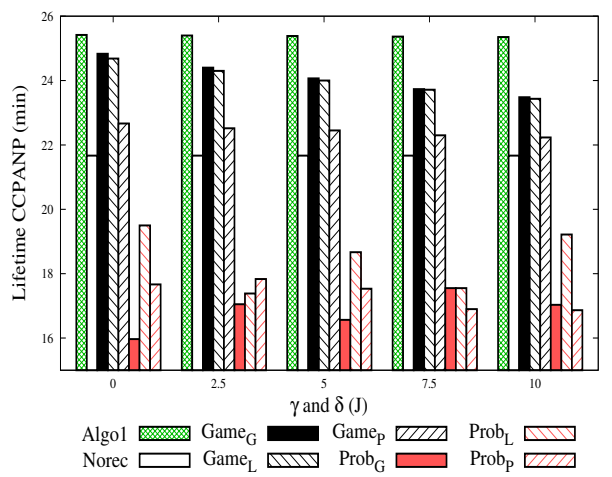

(b)

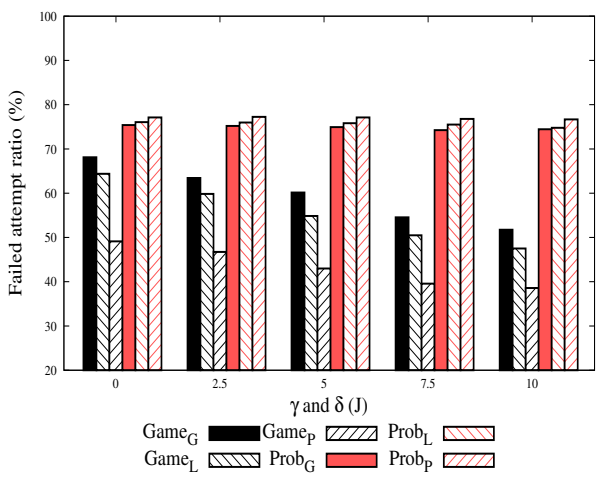

(c)

Fig. 7. The performance indexes as a function of $\gamma$ and $\delta$ (in the experiments $\gamma=\delta$ ). The lifetime is shown in Figure 7(a); the CCPANP lifetime is shown in Figure 7(b); the Failed attempt ratio is shown in Figure 7(c).

always perform worse than the basic method Norec algorithm, due to the impact of charging attempts, as also confirmed by Figure $7(\mathrm{c})$. In Figure 8 we show the $L_{\text {CCPANP }}$ index

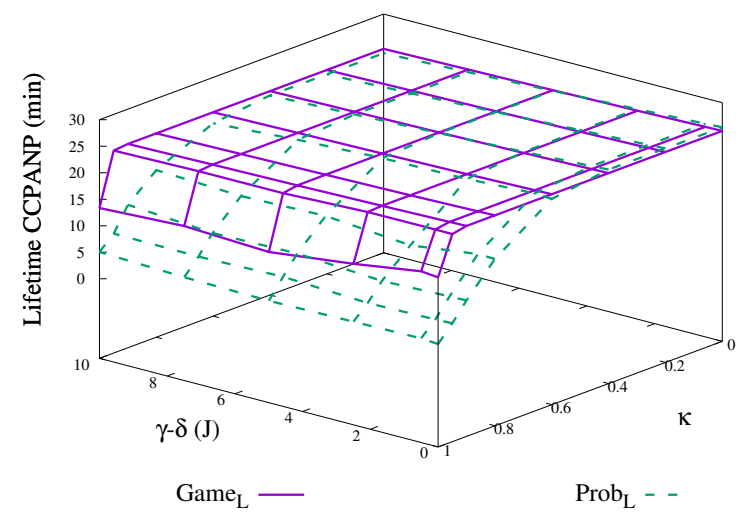

Fig. 8. The CCPANP lifetime index varying $\gamma-\delta$ and $\kappa$ for the methods Game $_{L}$ and $\operatorname{Prob}_{L}$.

when varying the $\kappa$ threshold (on the $x$ axis) and the $\gamma-\delta$ parameter (on the $y$ axis). We only compare the Game $_{L}$ and $\operatorname{Prob}_{L}$ algorithms, i.e. the game-theory based and probabilistic schemes both exploiting local cooperation among the UAVs. The Game $_{L}$ algorithm keeps the $L_{\text {CCPANP }}$ index very close to the optimal method, i.e. Algo1, dropping its performance only when requesting a continuous total coverage (i.e. $\kappa$ equal to 1 ). The $\operatorname{Prob}_{L}$ method, instead, starts reducing its performance from $\kappa=0.4$, and achieves much lower $L_{\text {CCPANP }}$ values than the Game $_{L}$ scheme for $\kappa \geq 0.8$. In Figure 9, we show the $L_{\mathrm{CCPANP}}$ index when varying the $\alpha$ and $\beta$ parameters, while keeping constant the values of $\gamma=\delta=5 J$. Again, we compared the Game $_{L}$ and $\operatorname{Prob}_{L}$ algorithms. In both cases, the optimal value is achieved when $\beta>\alpha$, as expected. However, the Game $_{L}$ scheme exploits much more efficiently the presence of the recharging station than the $\operatorname{Prob}_{L}$ algorithm, for both different recharge powers $(\beta)$ and flight discharge characteristics $(\alpha)$.

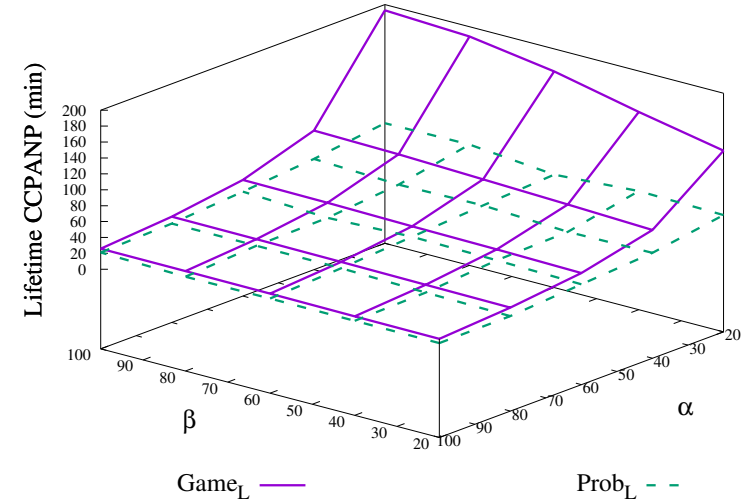

Fig. 9. The CCPANP lifetime index varying $\alpha$ and $\beta$ for the methods Game $_{L}$ and $\operatorname{Prob}_{L}$.

\section{Cooperation analysis}

Finally, we analyze the impact of UAV cooperation rate on the system performance, by considering the interval $T_{\text {STRATEGY }}$ in exchanging the STRATEGY broadcast messages. We recall from Section V-B that the STRATEGY messages contain the $E\left(a_{i}, t_{j}\right)$ values, needed to compute the mixed strategies in the game-theory distributed schemes as well as the charging probabilities in the distributed probabilistic schemes. The no-cooperation schemes, i.e. Game $_{P}$ and $\operatorname{Prob}_{P}$, are clearly not affected by the analysis and, hence, perform in the same way for all values of $T_{\text {STRATEGy. }}$ In Figures 10(a), 10(b) and 10(c) we depict the performance for the $L_{\text {final }}, L_{\text {CCPANP }}$ and $F_{\text {ratio }}$ indexes, over increasing values of $T_{\text {STRATEGY. }}$. We can notice that the game-theory based approaches keep good performance and are only slightly affected by the freshness of information coming from the other UAVs. This is a quite relevant result, since it shows that the network communication overhead can be reduced without impacting the $L_{\text {CCPANP }}$ performance, although the amount of energy drained by the communication module can represent only a small fraction of the energy drained by the rotors [15][16]. Vice-versa, the performances of probabilistic 


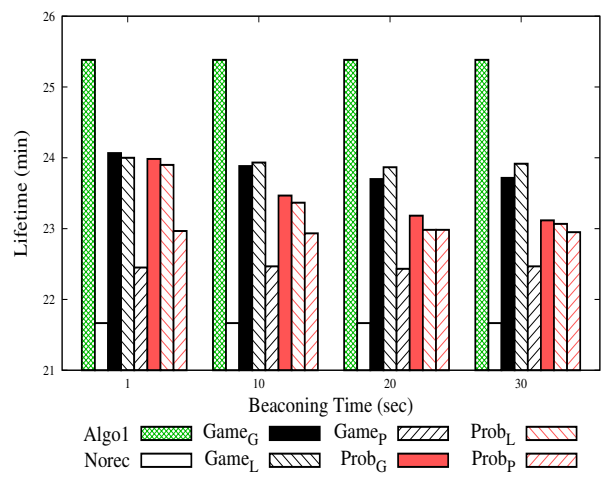

(a)

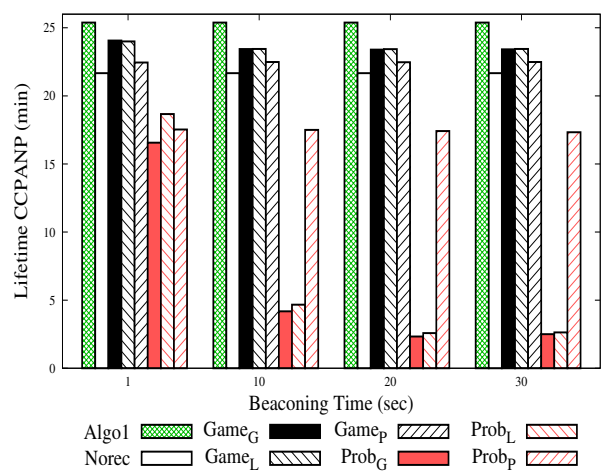

(b)

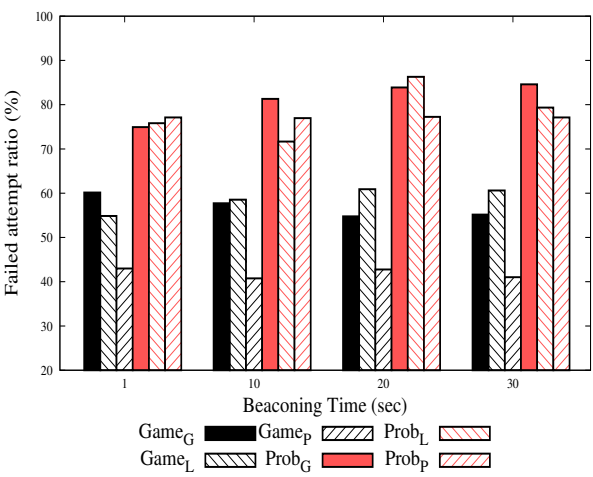

(c)

Fig. 10. The performance indexes as a function of the broadcast frequency of the STRATEGY message. The lifetime is shown in Figure 10(a); the CCPANP lifetime is shown in Figure 10(b); the Failed attempt ratio is shown in Figure 10(c).

approaches $\left(\operatorname{Prob}_{G}\right.$ and $\left.\operatorname{Prob}_{L}\right)$ decrease quite significantly when increasing the $T_{\text {STRATEGY }}$ interval. Hence, they need much higher communication overhead than the game-theory based algorithms.

\section{CONCLUSION}

In this paper, we have investigated the deployment of aerial mesh networks meeting requirements of scenario coverage and service continuity through scheduling and UAVs for groundbased recharging. We have developed the optimal solution in presence of a centralized controller as well as distributed deployment strategies based on game theory techniques and swarm mobility algorithms; different variants of the charging scheduling policies have been proposed according to the extent of cooperation among the UAVs. Our simulation results show that the distributed game-theory based solutions based on 1hop neighbor messaging outperforms probabilistic approaches, and performs close to the optimal solution, but without the overheads of central coordination and global cooperation. Our future work will extend the theoretical framework to multiple ground-charging stations, consider mobility of these stations, and investigate different altitude values for different UAVs.

\section{ACKNOWLEDGEMENTS}

This work was supported by the ALMAIDEA UniBO Grant Senior Project: "BeeDrones: environmental monitoring systems based on ultra-low power sensor nodes and aerial communications".

\section{APPENDIX A}

\section{PROOF OF LEMMA 1}

We observe that the Algorithm 1 keeps iterating the ROUND_ROBIN_STAGE till the following condition becomes true (line 40):

$$
E(*, k)<\alpha_{t} \cdot\left(N_{s}-1\right)+O P(h)
$$

where $E(*, k)$ is the energy of max Node (i.e. of the UAV with maximum residual energy), after having completed $k$ iterations in ROUND_ROBIN_STAGE mode. Moreover, at each iteration $k$, the following recursive property holds:

$$
E(*, k)=E(*, k-1)-x_{k} \cdot\left(\alpha_{t} \cdot\left(N_{s}-1\right)-\beta_{t}\right)-O P(h)
$$

where $x_{k}$ is the number of charging slots assigned to each UAV (i.e. the numRoundsPer $U A V$ at line 49, assuming extraSlots $=0$ ). By construction, $x_{k}$ is always equal to $\left\lfloor\frac{E(*, k-1)-O P(h)}{\alpha_{t} \cdot\left(N_{s}-1\right)}\right\rfloor$. Hence, (40) can be re-written into:

$$
E(*, k)=\psi \cdot(E(*, k-1)-O P(h))
$$

where $\psi=\frac{\beta_{t}}{\alpha_{t} \cdot\left(N_{s}-1\right)}$. By substituting (41) into (39) and iterating over $k$, we get the following condition:

$\psi^{k} \cdot\left(E_{\text {init }}-O P(h)+\frac{O P(h)}{1-\psi}\right)-\frac{O P(h) \cdot \psi}{1-\psi}<\alpha_{t} \cdot\left(N_{s}-1\right)$

Since the ROUND_ROBIN_STAGE ends as soon as the condition above becomes true, we derive the value of $k$ solving the equation. Let $K$ be such value. After some calculations (not reported here for space reasons), we obtain the expression of $K$ reported in (8).

\section{APPENDIX B \\ PROOF OF THEOREM 1}

In order to compute $T_{\mathrm{RR}}$, we consider the term $x_{\mathrm{RR}}=$ $\sum_{i=0}^{K-1} x_{i}$, which is the total number of charging slots assigned to each UAV during the ROUND_ROBIN_STAGE. From (41) we can derive the energy at step $k$ :

$$
E(*, k)=\psi^{k} \cdot E_{\text {init }}-O P(h) \cdot \frac{\psi-\psi^{k+1}}{1-\psi}
$$

Through (43) and the definition of $x_{k}$, we can derive $x_{\mathrm{RR}}$ as:

$$
\begin{aligned}
x_{\mathrm{RR}} & =\left\lfloor\sum_{k=0}^{K-1} \frac{(E(*, k)-O P(h))}{\alpha_{t} \cdot\left(N_{s}-1\right)}\right\rfloor \\
& =\left\lfloor\sum_{k=0}^{K-1} \frac{\left(\psi^{k} \cdot E_{\text {init }}-O P(h) \cdot \frac{\psi-\psi^{k+1}}{1-\psi}-O P(h)\right)}{\alpha_{t} \cdot\left(N_{s}-1\right)}\right\rfloor \\
& =\left\lfloor\frac{\left(E_{\text {init }}+\frac{O P(h) \cdot \psi}{1-\psi}\right) \cdot\left(1-\psi^{K}\right)-K \cdot O P(h)}{\alpha_{t} \cdot\left(N_{s}-1\right) \cdot(1-\psi)}\right\rfloor
\end{aligned}
$$


Assuming that all the $N_{s}$ UAVs will recharge of the same energy amount (i.e. extraRounds will always be equal to 0), we can derive $T_{\mathrm{RR}}$ as follows:

$$
T_{\mathrm{RR}}=x_{\mathrm{RR}} \cdot N_{s}
$$

When entering the RECHARGE_MINIMUM_STAGE, the energy of max Node is lower than $\alpha_{t} \cdot\left(N_{s}-1\right)$. Since each active node will discharge of $\alpha_{t}$ energy units at each slot, we have that at most $N_{s}-1$ can be completed till one UAV will drain its energy, hence: $0 \leq T_{\mathrm{MIN}}<N_{s}-1$. Combining this result with (44), we have the statement of Theorem 1.

\section{APPENDIX C PROOF OF COROLLARY 1}

The proof is derived from Lemma 1. At each iteration of the RECHARGE_ROBIN_STAGE, all the $N_{s}$ UAVs enter the charging state exactly once. Since this stage is iterated $K$ times (Lemma 1), the number of swaps is exactly equal to $K \cdot N_{s}$. Vice versa, in the RECHARGE_MINIMUM_STAGE, a swap can occur at each slot, since the minimum UAV is selected. Since $T_{\text {MIN }}<N_{s}-1$, we have that the total number of swaps is upper bound by $K \cdot N_{s}+N_{s}-1$.

\section{APPENDIX D}

\section{PROOF OF THEOREM 2}

We observe that the network lifetime is maximized when all the UAVs discharge at the same rate, i.e. when the difference between the energy of the most charged and least charged UAVs is minimized. We indicate with $\Delta$ such difference. Let $M I N S C H E D$ a scheduler selecting the minimum energy node at each slot $j$ (i.e. $s\left(a_{i}, t_{j}\right)=1$ if $i=$ getMinEnergyNode() $\forall j$ ). We notice that $M I N S C H E D$ is optimal in terms of maximum lifetime, and that $\Delta \leq \alpha_{t}+\beta_{t}$. We now prove that such a condition on $\Delta$ is also guaranteed by Algorithm 1. In the RECHARGE_MINIMUM_STAGE, our Algorithm follows the MINSCHED policy, hence the condition is always satified at each slot. In the RECHARGE_ROBIN_STAGE, the condition might not hold at each slot. However, we notice that in the allocateRoundCharge method, the difference of roundSize (i.e. of charging slots) between two UAVs is at most equal to one. This implies that, at the end of each iteration, we still have that $\Delta \leq \alpha_{t}+\beta_{t}$.

\section{APPENDIX E \\ PROOF OF THEOREM 3}

By Theorem 1, we show that the lifetime is maximized when charging operations are scheduled according to a round robin policy. Let roundSize $[i, j]$ be the duration of the charge -in terms of number slots- for UAV $i$ at iteration $j$. In Algorithm 1, roundSize $[i, j]$ is computed according to the allocateRoundCharge method. By absurd, let MINSWAP be another scheduler providing a number of swaps lower than $N_{s} \cdot K$, but guaranteeing the same lifetime than Algorithm 1. Since at each iteration the number of swaps is constant, and equal to $N_{s}$, we deduce that $M I N S W A P$ performs less iterations than Algorithm 1, which implies that for a given $k$, roundSize $[i, k]_{\text {MINSWAP }} \geq \operatorname{roundSize}[i, k]_{\text {Algorithm } 1}, \forall i$.
However, this is not possible, since by construction, Algorithm 1 computes the maximum duration of roundSize $[i, k]$ so that the last UAV going to recharge at iteration $k$ will not drain its battery before the end of the iteration.

\section{APPENDIX F \\ PROOF OF THEOREM 4}

We assume that $N_{S}$ is always greater then $N_{\min }+1$, where $N_{\text {min }}$ is defined in (7). At each time slot $t_{j}<t_{\text {final }}$ we have always $N_{s}-1$ UAVs in state $s_{\mathrm{fly}}$ and $1 \mathrm{UAV}$ in state $s_{\mathrm{rec}}$ (lines 7-21 of Algorithm 1). Since the centralized approach places the UAVs in an hexagonal pattern, each UAV $a_{i}$ being in state $s_{\text {fly }}$ at time slot $t_{j}$ uniquely covers at least a surface of:

$$
\operatorname{Surf}\left(t_{j}, a_{i}, h, \theta\right) \geq\left(h \cdot \tan \left(\frac{\theta}{2}\right)\right)^{2} \cdot \frac{3 \cdot \sqrt{3}}{2}
$$

With this result, we can rewrite (6) as follow:

$$
C\left(t_{j}\right) \geq \operatorname{Surf}\left(t_{j}, a_{i}, h, \theta\right) \cdot\left(N_{S}-1\right)
$$

and hence we have that $\rho_{j} \geq \kappa, \forall t_{j}<t_{\text {final }}$.

\section{APPENDIX G \\ PROOF OF THEOREM 5}

Let us consider the values of $p_{B}^{i, j}$ defined by (12) and $p_{R}^{k, j}$ as known. From (13) we can derive a system of $N_{S}-1$ equations with $N_{S}-1$ unknown variables, i.e. the $p_{G}^{i, j}$ variables, with $1 \leq i \leq N_{S}, i \neq k$. We have $\forall t_{j} \in T$ :

$$
\left\{\begin{array}{l}
p_{B}^{1, j}=1-\left(p_{R}^{k, j} \cdot \prod_{a_{h} \in A \backslash\left\{a_{1}\right\}}\left(1-p_{G}^{h, j}\right)\right) \\
p_{B}^{2, j}=1-\left(p_{R}^{k, j} \cdot \prod_{a_{h} \in A \backslash\left\{a_{2}\right\}}\left(1-p_{G}^{h, j}\right)\right) \\
\cdots \\
p_{B}^{N_{S}, j}=1-\left(p_{R}^{k, j} \cdot \prod_{a_{h} \in A \backslash\left\{a_{N_{S}}\right\}}\left(1-p_{G}^{h, j}\right)\right)
\end{array}\right.
$$

If $\phi\left(t_{j-1}\right)=1$ then $p_{R}^{k, j}=1$, hence we have again a system of $N_{S}$ equations with $N_{S}$ unknown variables. The solution of such system is the one presented in the Theorem 5 (see (14)). We can prove it by construction, i.e. by substituting (14) into any equation of the system of equations (47), verifying:

$p_{B}^{i, j}=1-\left(p_{R}^{k, j} \cdot \prod_{a_{h} \in A \backslash\left\{a_{i}\right\}} \sqrt[\left(N_{S}-1\right)]{\frac{\prod_{a_{q} \in A \backslash\left\{a_{h}\right\}}\left(1-p_{B}^{q, j}\right)}{\left(1-p_{B}^{h, j}\right)^{N_{S}-2} \cdot p_{R}^{k, j}}}\right)$

We need to remark that (14) can become inconsistent for UAV $a_{i}$, i.e $p_{G}^{i, j}<0$, when the following condition holds:

$$
\prod_{a_{h} \in A \backslash\left\{a_{i}\right\}}\left(1-p_{B}^{h, j}\right)>\left(1-p_{B}^{i, j}\right)^{N_{S}-2} \cdot p_{R}^{k, j}
$$

In such case, we transform the mixed strategy in a pure strategy, i.e the support of the mixed strategy consists in a single action, and (14) is rewritten as follows:

$$
p_{G}^{i, j}=\operatorname{MAX}\left(0,1-\sqrt[\left(N_{S}-1\right)]{\frac{\prod_{a_{h} \in A \backslash\left\{a_{i}\right\}}\left(1-p_{B}^{h, j}\right)}{\left(1-p_{B}^{i, j}\right)^{N_{S}-2} \cdot p_{R}^{k, j}}}\right)
$$




\section{APPENDIX H \\ PROOF OF THEOREM 6}

Similar to the previous case, by substituting (14) in (20) we obtain:

$$
p_{T_{0}}^{k, j}=\prod_{a_{h} \in A \backslash\left\{a_{k}\right\}}\left(\sqrt[\left(N_{S}-1\right)]{\frac{\prod_{a_{q} \in A \backslash\left\{a_{h}\right\}}\left(1-p_{B}^{q, j}\right)}{\left(1-p_{B}^{h, j}\right)^{N_{S}-2} \cdot p_{R}^{k, j}}}\right)
$$

After some arithmetic calculus, it is easy to notice that (22) is a re-arrangement of (51).

As for the previous proof, we have to remark that the Equation above can become inconsistent for the UAV $a_{k}$, i.e $p_{R}^{k, j}>1$, if the following condition holds:

$$
\prod_{a_{h} \in A \backslash\left\{a_{k}\right\}}\left(1-p_{B}^{h, j}\right)>\left(p_{T_{0}}^{k, j}\right)^{N_{S}-2}
$$

In this case we transform the mixed strategy in a pure strategy, i.e the support of the mixed strategy is formed by only one action. More formally (22) is rewritten as follows:

$$
p_{R}^{k, j}=\operatorname{MIN}\left(1, \sqrt[\left(N_{S}-1\right)]{\frac{\prod_{a_{h} \in A \backslash\left\{a_{k}\right\}}\left(1-p_{B}^{h, j}\right)}{\left(p_{T_{0}}^{k, j}\right)^{N_{S}-2}}}\right)
$$

\section{REFERENCES}

[1] Valour consultancy. The Future of Commercial and Industrial UAVs. Brochure, 2016, available online at: http://www.valourconsultancy.com/wp-content/uploads/2015/12/TheFuture-of-UAVs-Drones-Report-Brochure.pdf

[2] B. D. Song, J. Kim, J. Kim, H. Park, J. R. Morrison and D. H Shim. Persistent UAV service: an improved scheduling formulation and prototypes of system components. Journal of Intelligent \& Robotic Systems: 74(1), pp. 221-232, 2014.

[3] H. Shakhatreh, A. Khreishah, J. Chakareski, H. B. Salameh and I. Khalil. On the continuous coverage problem for a swarm of UAVs. Proc. of IEEE Sarnoff Symposium, Princeton, USA, 2016.

[4] A. Trotta, M. Di Felice, K. Chowdhury and L. Bononi. Fly and Recharge: Achieving persistent coverage using small unmanned aerial vehicles (SUAVs). 2017 IEEE International Conference on Communications (ICC), Paris, France, 2017, pp. 1-7.

[5] M. Erdelj, E. Natalizio, K. R. Chowdhury and I. F. Akyildiz Help from the Sky: Leveraging UAVs for Disaster Management. IEEE Pervasive Computing: 16(1): 24-32 (2017).

[6] A. Osseiran et alt.. Scenarios for 5G mobile and wireless communications; the vision of the METIS project. IEEE Communication Magazine, 52(5), pp. 26-35, 2014.

[7] Y. Zeng, R. Zhang and T. Joon Lim. Wireless communications with unmanned aerial vehicles: opportunities and challenges. IEEE Cоттиnication Magazine, 54(5), pp. 36-42, 2016.

[8] M. Mozaffari, W. Saad, M. Bennis and M. Debbah. Efficient deployment of multiple unmanned aerial vehicles for optimal wireless coverage. IEEE Communication Letters, 20(8), pp.1647-1650, 2016.

[9] M. Di Felice, A. Trotta, L. Bedogni, K.R. Chowdhury and L. Bononi Self-Organizing Aerial Mesh Networks for Emergency Communication. 2014 IEEE 25th Annual International Symposium on Personal, Indoor, and Mobile Radio Communication (PIMRC), Washington DC, 2014, pp. $1631-1636$

[10] J. Yu, S. J. Chung and P. G. Voulgaris. Target Assignment in Robotic Networks: Distance Optimality Guarantees and Hierarchical Strategies. IEEE Transactions on Automatic Control, vol. 60, no. 2, pp. 327-341, Feb. 2015.

[11] K. Derr and M. Manic. Extended virtual spring mesh (EVSM): The distributed self-organizing mobile ad hoc network for area exploration. IEEE Trans. on Industrial Electronics: 58(12), pp. 5424-5437, 2011.

[12] M. Asadpour, B. Van den Bergh, D. Giustiniano, K. A. Hummel, S Pollin and B. Plattner. Micro aerial vehicle networks: an experimental analysis of challenges and opportunities. IEEE Communications Magazine: vol. 52, no. 7, pp. 141-149, July 2014.
[13] R. Shinkuma and Y. Goto. Wireless multihop networks formed by unmanned aerial vehicles with separable access points and replaceable batteries. 2016 IEEE 7th Annual Ubiquitous Computing, Electronics \& Mobile Communication Conference (UEMCON): New York, NY, 2016, pp. 1-6.

[14] J. Leonard, A. Savvaris and A. Tsourdos. Energy management in swarm of Unmanned Aerial Vehicles. 2013 International Conference on Unmanned Aircraft Systems (ICUAS): 2013, pp. 124-133.

[15] L. Gupta, R. Jain and G. Vaszkun. Survey of important issues in UAV communication networks. IEEE Communications Survey \& Tutorials: 18(2), pp. 1123-1152, 2016.

[16] D. Halperin, B. Greenstein, A. Sheth and D. Wetherall. Demystifying 802.11n power consumption. Proc of IEEE HotPower, Vancouver, Canada, 2010

[17] J. Kim, B. D. Song and J. R. Morrison. On the scheduling of systems of UAVs and fuel service stations for long-term mission fulfillment. Journal on Intelligent Robot Systems: 70(1), pp. 347-359, 2013.

[18] J. Scherer and B. Rinner. Persistent multi-UAV surveillance with energy and communication constraints. 2016 IEEE International Conference on Automation Science and Engineering (CASE), Fort Worth, TX, 2016, pp. 1225-1230.

[19] Federal Communication Commission (FCC). Deployable Aerial Communications Architecture in Emergency Communications. 2011.

[20] S. Morgenthaler, T. Braun, Z. Zhao, T. Staub and M. Anwander. UAVNet: a mobile wireless mesh network using unmanned aerial vehicles. 2012 IEEE Globecom Workshops, Anaheim, CA, 2012.

[21] M. Alzenad, A. El-Key, F. Lagum and H. Yanikomeroglu.. 3D placement of an unmanned aerial vehicle base station (UAV-BS) for energy-efficient maximal coverage. IEEE Communication Letters, 99(1), pp. 1-4, 2017.

[22] E. Yanmaz. Connectivity versus area coverage in unmanned aerial vehicle networks. 2012 IEEE International Conference on Communications (ICC), Ottawa, Canada, 2012, pp. 719-723.

[23] C. Di Franco and G. Buttazzo. Energy-aware coverage path planning of UAVs. 2015 IEEE International Conference on Autonomous Robot Systems and Competitions (ICARSC), Vila Real, Portugal, 2015.

[24] K. Daniel, S. Rohde, N. Goddemeier and C. Wietfield. Cognitive agent mobility for aerial sensor networks. IEEE Sensors Journal: 11(11), pp. 2671-2682, 2013.

[25] N. Nigam, S.Bicniawski, I. Kroo and J. Vian. Control of multiple UAVs for persistent surveillance: algorithm and flight test results. IEEE Transactions on Control System Technology, 20(5), pp. 1236-1251, 2012.

[26] C.-I. Vasile and C. Belta. An automata-theoretic approach to the vehicle routing problem. Robotics: Science and Systems: pp. 1-9, 2014.

[27] K. A. Swieringa, C. B. Hanson, J. R. Richardson, J. D. White, Z. Hasan, E. Qian and A. Girard. Autonomous battery swapping systems for smallscale helicopters. 2010 IEEE International Conference on Robotics and Automation (ICRA), Anchorage, USA, 2010.

[28] K. Yu, A. K. Budhiraja and P. Tokekar. Algorithms for Routing of Unmanned Aerial Vehicles with Mobile Recharging Stations and for Package Delivery. CoRR, abs/1704.00079, 2017.

[29] N. Mathew and S. L. Smith and S. L. Waslander. Multirobot Rendezvous Planning for Recharging in Persistent Tasks. IEEE Transactions on Robotics: 31(1), pp. 128-142, 2015.

[30] N. K. Ure, G. Chowdhary, T. Toksoz, J. P. How, M. A. Vavrina, and J. Vian. An automated battery management system to enable persistent missions with multiple aerial vehicles. IEEE/ASME Transactions on Mechatronics: 20(1), pp. 275-286, 2015.

[31] K. Dorling, J. Heinrichs, G. G. Messier, S. Magierowski Vehicle Routing Problems for Drone Delivery. IEEE Transactions on Systems, Man, and Cybernetics: Systems: 47(1), pp. 70-85, 2017.

[32] H. Zhang, Z. Zhang and H. Dai Gossip-Based Information Spreading in Mobile Networks. IEEE Transactions on Wireless Communications, vol. 12, no. 11, pp. 5918-5928, November 2013.

[33] S. Vasudevan, J. Kurose and D. Towsley Design and analysis of a leader election algorithm for mobile ad hoc networks Proceedings of the 12th IEEE International Conference on Network Protocols, 2004. ICNP 2004., 2004, pp. 350-360.

[34] B. Wang, H. Beng Lim, D. Ma. A survey of movement strategies for improving network coverage in wireless sensor networks. Computer Communications, 32(1), pp. 1427-1436, 2009.

[35] Osborne Martin J. An introduction to game theory. New York: Oxford university press, Vol. 3, No. 3, 2004.

[36] The OMNeT++ tool. Project website. http://omnetpp.org 


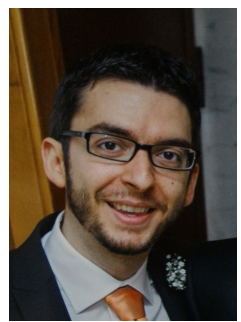

Angelo Trotta received his Master Degree (summa cum Laude) in Computer Science in 2011, from the University of Bologna, Italy. From October 2012 to October 2013 he was a Research Student for the Italian PRIN 2009 project STEM-Net working on the study, modeling and simulation of cognitive radio technologies and self-organizing wireless networks. In May 2017 he received the Ph.D. degree in Computer Science and Engineering from the University of Bologna, Italy. In 2015-2016, he was a visiting researcher at the Heudiasyc Laboratory, Sorbonne Universits, UTC, Compigne, France. In 2018, he was a visiting researcher at the Genesys-Lab, Northeastern University, Boston, MA, USA. Currently he is a post-doc Research Fellow at the Department of Computer Science and Engineering, University of Bologna, Italy, working on nature-inspired algorithms for self-organizing multi-robots wireless systems.

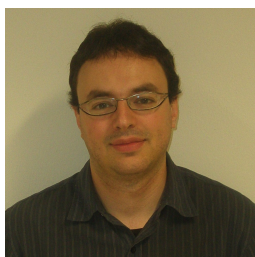

Marco Di Felice received the Laurea (summa cum laude) and Ph.D. degrees in computer science from the University of Bologna, Italy, in 2004 and 2008, respectively. In 2007, he was a visiting researcher with the Georgia Institute of Technology, Atlanta, GA, USA. In 2009, he was a visiting researcher with Northeastern University, Boston, MA, USA. Currently, he is an Associate Professor in computer science with the University of Bologna. His research interests include self-organizing wireless networks, unmanned aerial systems, IoT and mobile applications. Prof. Di Felice currently serves on the editorial board of Elseviers Ad Hoc Networks journal. He authored more than 80 papers on wireless and mobile systems, receiving the Best Paper Award at the Association for Computing Machinery International Symposium on Mobility Management and Wireless Access (MOBIWAC) in 2012 and at the IEEE Annual Mediterranean Ad Hoc Networking Workshop (MED-HOC-NET) in 2013.

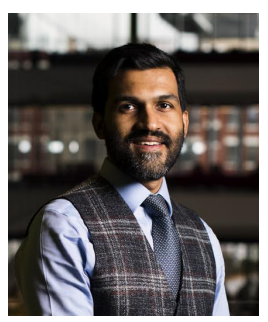

Kaushik R. Chowdhury (M09SM15) received the $\mathrm{PhD}$ degree from the Georgia Institute of Technology, Atlanta, in 2009. He is currently Associate Professor and Faculty Fellow in the Electrical and Computer Engineering Department at Northeastern University, Boston, MA. He was awarded the Presidential Early Career Award for Scientists and Engineers (PECASE) in Jan. 2017, the DARPA Young Faculty Award in 2017, the Office of Naval Research Director of Research Early Career Award in 2016, and the National Science Foundation (NSF) CAREER award in 2015. He received multiple best paper awards, including three in the IEEE ICC conference, in 2009, 12 and 13, and ICNC conference in 2013. He serves on the editorial board of the IEEE Transactions on Wireless Communications, His research has been supported by the NSF, Office of Naval Research, DARPA, MathWorks, among others. His current research interests are in dynamic spectrum access networks and systems, wearables and implant communication and energy harvesting sensors.

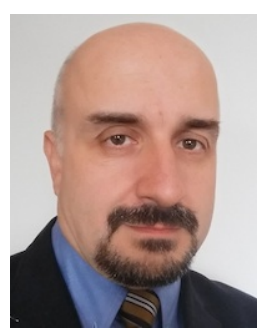

Luciano Bononi (MSC, Summa cum laude, 1997, Ph.D, 2001), is Associate Professor of Wireless and Mobile Systems, Mobile Applications and Internet of Things at the Department of Computer Science and Engineering of the University of Bologna. He has co-authored more than 110 peer reviewed conference and journal publications and 8 book chapters, receiving three best paper awards, and his research areas include wireless systems and networks, protocol architectures, Internet of Things, Internet of Energy, modeling, simulation, performance evaluation, mobile services and mobile applications. He has been involved in more than 10 international research projects, and he is Associate Editor of five international Journals and guest edited more than 10 special issues. He was chair in more than 15 IEEE/ACM conferences and TPC member in more than 140 IEEE/ACM conferences on the above research topics. He is the founder and director of the Laboratory of Wireless Systems and Mobile Applications at CSE.

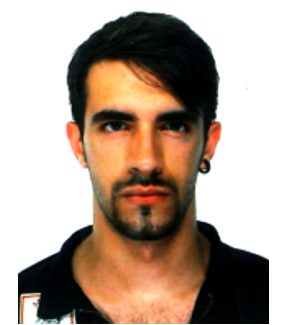

Federico Montori received his Master Degree (summa cum Laude) in Computer Science in 2015 from the University of Bologna, Italy. He worked in the European projects Internet of Energy (from 2012 to 2013) and Arrowhead (from 2014 to 2016) within the scope of IoT platform interoperability in the field of smart energy. In 2015 he held a research grant from the Advanced Research Center on Electronic Systems (ARCES) and has been a visiting researcher at Luleå University of Technology, Sweden. Currently he is a $\mathrm{PhD}$ student at the Department of Computer Science and Engineering, University of Bologna, Italy, working on algorithms for Mobile Crowdsensing (MCS) and architectures for the collaborative IoT (C-IoT) within the scope of Smart Cities and environmental monitoring. 